\title{
3. Topographie und Architektur der Komnenen-Mauer
}

\subsection{Die Errichtungszeit der Komnenen-Mauer}

Auf die Mauerabschnitte in den unteren Blachernen (Abb. 35) wurden bereits oben eingegangen (siehe Abschnitt 2.1.1.), da die Frage ihrer Bauphasen unmittelbar mit dem Problem der nördlichen Ausdehnung der Theodosianischen Landmauer verknüpft war. Im folgenden wird die Strecke zwischen Tekfur Sarayı bis zu den Substruktionen behandelt, die westlich der Mumhane-Mauer verläuft. Der Mauerabschnitt zwischen dem nördlichen Turm von Tekfur Sarayı (im folgenden Tekfur Sarayı-Nordturm) und Turm 12 der Blachernen-Mauer wird seit dem 19. Jahrhundert Kaiser Manuel I. Komnenos (1143-1180) zugeschrieben und als Manuel-Mauer bzw. Komnenen-Mauer bezeichnet (Abb. 33, 34, 147-158, 161). ${ }^{516}$ Die von Niketas Choniates erwähnte Mauer, die der Kaiser „zum Schutze des Blachernen-Palastes“ aufgeführt habe, ${ }^{517}$ entspricht nämlich dieser Mauerlinie, zumal Niketas später auch über die Lage der Mauer indirekt Auskunft gibt. ${ }^{518}$ Doch weder die Architektur noch das Mauerwerk der gesamten Wehranlage sind homogen. Während die ersten acht Türme des Südtrakts halbrund, hufeisenförmig bzw. polygonal und recht stattlich sind (Abb. 19, 147, 149), wurden die Türme 9 bis 12 rechteckig und abgesehen vom Turm 9 (Abb. 150) - in etwas kleineren Dimensionen errichtet (Abb. 33, 148, 152). ${ }^{519}$ Turm 9 wird sowohl auf Grund seiner Größe als auch wegen seines Mauerwerks den ersten acht Türmen zugerechnet. Im folgenden wird der Abschnitt von Tekfur Sarayi-Nordturm bis einschließlich Turm 9 als Südtrakt, der Abschnitt von Turm 9 bis einschließlich Turm 12 als Nordtrakt bezeichnet. Die Kurtinen sind ebenfalls verschiedenartig ausgebildet, indem die stadtseitigen Nischen des Wehrgangs des Südtrakts in der Regel breiter sind (Breite am Nordtrakt ca. 1,90 m, an der Kurtine 11/12 aufgenommen, Abb. 154; Breite am Südtrakt ca. 2,30 m, an der Kurtine 2/3 aufgenommen,

516 Die richtige Identifizierung der Mauer erfolgte durch Mordtmann, Esquisse, 11-12, und durch Van Millingen, Walls, 122-127; siehe auch Meyer-Plath/Schneider, Landmauer, 102 (Schneider), 109-110 (Meyer-Plath); Müller-Wiener, Topographie, 301305.

517 Niketas Choniates, 384/35-38; deutsche Übersetzung: Grabler, Abenteurer, 184-185.

518 Niketas Choniates, 543/95-7; deutsche Übersetzung: Grabler, Kreuzfahrer, 118.

519 Vgl. Meyer-Plath/Schneider, Landmauer, 110, Abb.27. - Für eine kurze Abhandlung über die Mauertechnik der Komnenen-Mauer siehe Schneider/Meyer, Zweiter Vorbericht, 14-15 (Meyer). 
Abb. 153) ${ }^{520}$ Ein weiterer augenfälliger Unterschied besteht in der Mauertechnik. Das Mauerwerk ändert sich unmittelbar nach Turm 9 (Abb. 21, 150) (zu den späteren Reparaturen siehe Abschnitt 3.2.). Alle diese Merkmale werfen die Frage auf, ob die gesamte Mauerlinie in ihrer heutigen Gestalt aus einer einzigen Bau- bzw. Planungsphase stammen kann. Van Millingen notierte zwar auf seinem Sketch Plan (Abb. 34), daß es sich bei der Mauer ab Turm 9 um palaiologische Reparaturen handelt, ging aber im Textteil nicht näher darauf ein. ${ }^{521}$

Meyer-Plath/Schneider wiesen ebenfalls auf die Unterschiede des südlichen und nördlichen Trakts hin und datieren den Südtrakt in das Jahr $1150 .{ }^{522}$ Foss begründete das andersartige Aussehen der Mauer ab Turm 9 folgenderweise: ${ }^{523}$

These walls are of such strikingly different appearance that they were formerly supposed to belong to widely separated periods. Good evidence exists, however, to show that they were contemporary. First, the northern walls continue the line of the southern and in no place constitute an obvious rebuilding of earlier walls. If they were of much later date, the defences of the Blachernae would simply have stopped at mid-point, leaving a large area unprotected. Further evidence comes from the wall which joins the first tower of Manuel with the Tekfur Saray. For most of its course, it has almost identical with the northern wall, using brick bands separated by alternating rows of stone and brick.

Die Errichtung der Wehranlage kann selbstverständlich nicht irgendwo in der Mitte aufgehört haben, doch ist es denkbar, daß ein Teil der Mauer später unfangreich repariert bzw. wiederhergestellt werden mußte, weswegen sein Aussehen von anderen abweicht. Die scheinbare Ähnlichkeit zwischen dem Mauerwerk des nördlichen Trakts (Abb. 148) und des Abschnitts Tekfur SarayıNordturm bis Turm 1 (Abb. 20) bedarf natürlich einer Erklärung. Um einen besseren Überblick gewinnen zu können, soll das Mauerwerk bzw. das Baumaterial der Komnenen-Mauer im folgenden kurz skizziert werden:

1) Unregelmäßige große rechteckige bzw. quadratische Quader (55 x 60 bis 80 x $50 \mathrm{~cm}$ - soweit ich sie probeweise vermessen konnte) werden von siebenbzw. neunschichtigen Ziegelbändern unterbrochen (Mauerprobe: Ziegelbandhöhe ca. $62 \mathrm{~cm}$, Ziegelbreite ca. 29 bis $35 \mathrm{~cm}$, Ziegelhöhe ca. 2,5, 3,5

520 Vgl. Meyer-Plath/Schneider, Landmauer, 109 (Meyer-Plath). - Dieser Abschnitt ist bei Müller-Wiener, Topographie, 306, Abb. 350a, mit der stadtseitigen Kurtine 12/13 verwechselt.

521 Van Millingen, Walls, Sketch Plan of the Blachernae Quarter, gegenüber 41. - Janin, Constantinople, 283, notierte: „On ne sait si la portion de muraille marquée par les quatre tours carrées qui flanquent les murs à l'endroit où ils descendent vers la Corne d'Or appartiennent au mur de Manuel Comnène."

522 Meyer-Plath/Schneider, Landmauer, 25 (Meyer-Plath). - Eine genauere Datierung des Nordtrakts geht daraus nicht hervor.

523 Foss, Fortifications, 57. 
bzw. $4 \mathrm{~cm}$, Mörtelhöhe ca. 4 bis $6 \mathrm{~cm}$ ). Während in den unteren Bereichen großformatige Quader verwendet worden sind, werden die Quader aufwärts kleiner. Dieses Mauerwerk, das nur am Südtrakt begegnet, wird im folgenden mit dem Buchstaben A bezeichnet (Abb. 149, 150). An den stadtseitigen Kurtinen wurden kleinere Quader verwendet (Abb. 153, 157). ${ }^{524}$ Das Mauerwerk tritt hin und wieder in Verbindung mit verdeckter Schichttechnik auf. Es enthält auch Holzanker.

2) Unregelmäßige Quader mit Ziegeldurchschuß (ein- bis dreilagig) und achtbis neunlagigen Ziegelschichten. Dazwischen treten auch vier Ziegelschichten in verdeckter Schichttechnik in Erscheinung (Abb. 148, 151, 152). ${ }^{525}$ Dieses Mauerwerk wird mit dem Buchstaben B bezeichnet. Es kommt an den Türmen und Kurtinen des Nordtrakts vor (Mauerprobe im Bereich der verdeckten Schichttechnik: Ziegelbandhöhe 63 bzw. $80 \mathrm{~cm}$, Ziegelbreite $30 \mathrm{~cm}$, Ziegelhöhe ca. 3 bis 3,5 bzw. $5 \mathrm{~cm}$, Mörtelhöhe 11 bzw. $12 \mathrm{~cm}$ ). Holzanker sind auch hier anzutreffen.

3) Unregelmäßige Quader (in der Regel vier Lagen) mit Ziegeldurchschuß in allen Lagefugen (ein bis drei) und sieben- bis achtschichtige Ziegelbänder. Schmale Ziegel (2 bis 2,5 cm) überwiegen. Verdeckte Schichttechnik kommt nicht vor. Dieses Mauerwerk wird mit dem Buchstaben $C$ bezeichnet (Abb. 20). Er ist an der angebauten Treppe hinter Turm 8 (Abb. 155) und an der stadtseitigen Kurtine 9/10 (Abb. 21) ${ }^{526} \mathrm{zu}$ finden (Mauerprobe von der Treppe: Ziegelbandhöhe $63 \mathrm{~cm}$, Ziegelbreite 28 bis $30 \mathrm{~cm}$, Ziegelhöhe 2 bis $2,5 \mathrm{~cm}$, Mörtelhöhe 3 bis $5 \mathrm{~cm}$ ). Die Kurtine zwischen Tekfur SaraylNordturm bis Turm 1 zeigt teilweise ähnliches Mauerwerk wie das stadtseitige Mauerwerk des Bereichs bis Turm 9. Besonders an beiden stadtseitigen Nischen an der Südseite von Turm 1 (Abb. 20) sind folgende Werte zu beobachten: Ziegelbandhöhe $57 \mathrm{~cm}$, Ziegelbreite 29 bis $34 \mathrm{~cm}$, Ziegelhöhe 2,5 bzw. $4 \mathrm{~cm}$, Mörtelhöhe 3,5 bzw. $4 \mathrm{~cm}$. Die Mauer steht hier mit dem Turm im Verband. Ab der zweiten Nische bis zum Tekfur Sarayl-Nordturm entspricht das Mauerwerk dem Typus C. ${ }^{527}$

524 Die stadtseitigen Kurtinen sind an mehreren Abschnitten auf Grund der intensiven Bebauung heute nur schwer zugänglich.

525 Wichtigste Literatur zur verdeckten Schichttechnik: Brunov, Hofbaukunst, 233; Schneider, Byzanz, 13-14; Mango, Narthex Mosaics, 249-250; Hallensleben, Pammakaristos, 154-156, 159-161, 163, 166, Anm. 102; Ötüken, İsa Kap1, 105-117; Vokotopoulos, Concealed Course, 247-260; Ousterhout, Observations, 163-170; ders., Master Builders, 174-175.

526 Es wird im allgemeinen angenommen, daß bereits an dieser Kurtine ähnliches Mauerwerk wie am Nordtrakt vorkommt, siehe Foss, Fortifications, 57-58. Das Mauerwerk unterscheidet sich aber von den Mauern des Nordtraktes, da hier vor allem keine verdeckte Schichttechnik zu erkennen ist.

527 Foss, Fortifications, 57, vergleicht das Mauerwerk der Kurtine mit dem Mauerwerk des Nordtrakts. Das Mauerwerk unterscheidet sich von diesem, indem hier überwiegend 
4) Kleine unregelmäßige Quader ohne Ziegeldurchschuß. Das Mauerwerk wird in den folgenden Abschnitten mit dem Buchstaben D bezeichnet. Dieses Mauerwerk kommt in den oberen Bereichen der Türme und Kurtinen sowie an Kurtine 12/13 vor (Abb. 160, 162).

Im folgenden Abschnitt wird zunächst Mauerwerk A und B berücksichtigt. Foss konzentrierte sich ebenfalls auf A und B und erklärte die Unterschiede folgendermaßen $:^{528}$

Perhaps the masons of the northern section simply ran out of large spoils and begun to use the smaller stones which needed levelling courses; or the gang working in the north had access to fewer ruined buildings; or the northern walls were separated by a sufficient lapse of time from the southern (though both still dating from the long reign of Manuel) that changes in aesthetic permitted what had been hidden to be displayed.

Es ist theoretisch möglich, daß die Mauertechnik infolge des Ausgehens der großen Quader geändert werden mußte, was als Grund für das unterschiedliche Aussehen der beiden Trakte angeführt werden könnte. ${ }^{529}$ Doch scheint mir die Möglichkeit plausibler, daß die gesamte Mauer zwar gleichzeitig errichtet wurde, aber ein Trakt aus irgendeinem Grund später neu aufgeführt werden mußte, worauf - soweit mir bekannt - in der Literatur noch niemals detailliert eingegangen wurde. Es würde also bedeuten: Entweder ist die KomnenenMauer im ursprünglichen Zustand von Tekfur Sarayi-Nordturm bis Turm 12 (bzw. noch etwas weiter nördlich) einheitlich verlaufen und sah wie der heutige Südtrakt aus, d.h. mit großen Türmen, die in der Mauertechnik A erbaut waren, wobei zu einem unbekannten Zeitpunkt der Nordteil abstürzte und in einer abweichenden Mauertechnik (B) repariert wurde. Oder die gesamte Komnenen-Mauer sah ursprünglich wie der heutige Nordtrakt aus, d.h. mit etwas kleineren Türmen, die im Mauerwerk B errichtet waren. In diesem Falle wäre der Südtrakt eingestürzt und wurde mit einem abweichenden Mauerwerk (A) neu aufgeführt.

Es stellen sich jedoch die folgenden Fragen: Wann wurde die KomnenenMauer überhaupt erbaut, welche Ursachen können die Reparatur bzw. die Wiederherstellung veranlaßt haben und welcher zeitliche Abstand könnte dann zwischen den beiden Teilen liegen? Ohne ausreichende Argumente wurden die Mauern teils in die frühere, teils in die spätere Phase der fast vierzigjährigen

dünne Ziegel vorkommen und, wie der Autor zugibt, verdeckte Schichttechnik nicht in Erscheinung tritt.

528 Foss, Fortifications, 57.

529 Wir wissen aus dem Eparchenbuch, das die Situation des 10. Jahrhunderts schildert, daß während der Bauarbeiten Knappheit an Baumaterial auftreten konnte; siehe Koder, Eparchenbuch, 139/22.1. 
Regierungszeit Kaiser Manuels I. Komnenos datiert. ${ }^{530}$ Da Niketas Choniates in zwei Passagen ganz klar ausdrückt, daß die Mauer „zum Schutz des BlachernenPalastes“ errichtet worden sei, ${ }^{531}$ kann angenommen werden, daß der Mauerbau gleichzeitig mit der intensiven Bautätigkeit des Kaisers im Blachernen-Palast erfolgte. Über die Bautätigkeit im Blachernen-Palast gewinnen wir für die frühere komnenische Zeit aus den Quellen nur spärliche Nachrichten. Wir erfahren lediglich, daß bereits zur Zeit des Kaisers Alexios I. Komnenos (10811118) hier ein Triklinos (Thronsaal) errichtet wurde. ${ }^{532}$ Ob Ioannes II. Komnenos (1118-1143) innerhalb seiner fünfundzwanzigjährigen Regierungszeit ebenfalls am Blachernen-Palast gebaut hat und in welchem Palast er überwiegend residierte, entzieht sich unserer Kenntnis. Den beiden oben erwähnten Passagen des Niketas Choniates ist zu entnehmen, daß Manuel zu diesem Palast eine besonders enge Beziehung hatte. ${ }^{533}$ Für seine erste Frau Bertha von Sulzbach (Eirene) ließ Manuel ein Gebäude errichten, das Niketas Choniates als das „hochbedachte Gebäude, welches Polytimos genannt wird“ bzw. als das „hohe Haus der alamanischen Herrin“ bezeichnete. ${ }^{534}$ Es wird daher angenommen,

530 Siehe Anm. 522. - Foss, Fortifications, 47 und 58, vertrat die Meinung, daß die Mauer einer späteren Periode des Kaisers Manuel bzw. dem späteren 12. Jahrhundert angehört; ebd., 56, wird die Mauer ca. 1160 datiert.

531 Vgl. Anm. 517 und 518.

532 Magdalino, Great Palace, 106-108; vgl. auch Tinnefeld, Blachernenpalast, 278.

533 Noch zur Zeit Manuels war der Große Palast nicht aufgegeben; im Jahre 1166 trat hier ein Konzil zusammen, siehe Mango, Edict, 317-330, bes.320; siehe auch Magdalino, Great Palace, 101-114. - Kurz vor der Myriokephalon-Schlacht (1176) muß auch innerhalb des Großen Palastes ein Muchrutas genannter Schaupavillion errichtet worden sein, siehe dazu Asutay-Effenberger, Muchrutas, 313-329.

534 Niketas Choniates, 271/43-44 und 544/12-13; deutsche Übersetzung: Grabler, Abenteurer, 62, und ders., Kreuzfahrer, 118. - Villehardouin, Conquête, 104, $\$ 182$, spricht ebenfalls von einem hohen Palast: „Si le vestent emperialment; si l'emporterent el halt palais de Blacquerne ... "Für weitere Quellen siehe Schreiner, Stadt ohne Menschen, 133 (Benjamin von Tudela, 1165/1170); Tinnefeld, Blachernenpalast, 277-278 (Odo von Deuil, 1147). - Schneider, Blachernen, 100, Anm. 1, zufolge erhob sich das Gebäude über den Substruktionen hinter dem Isaakios Angelos-Turm; ebd. Anm.2, notierte er: „Ich habe zwar Hypsela über den Substruktionen nördlich der İvaz Efendi angenommen, gerade so gut könnten sie aber auch auf der $7 \mathrm{~m}$ hohen, durch den Mauerzug westlich Çeşme Nr. 4 [Çınar Çeşme Sokak, heute Ağaçlı Çeşme Sokak] abgestürzten Anhöhe gelegen haben. Der Quartiername Lonca (= loggia) könnte andeuten, daß über der dahinterliegenden Mauer balkonartige Säulengänge zu sehen waren." Es soll hier notiert werden, daß, wie aus den Stiftungsurkunden Mehmets II. hervorgeht, man früher unter „Lonca“ auch Substruktionen verstanden hat; siehe Ergin, Vakfiye, 233, \$196. Später wurde unter „Lonca“ die Organisation bestimmter Gruppen von Gewerbetreibenden und Handwerkern (Esnaf) bzw. ihr Versammlungsort (Esnaf Loncası) verstanden; siehe Loncalar, Osmanlı Dönemi, in: IA 5 (1994) 225-226. In diesem Gebiet befindet sich eine große Straße, die „Esnaf Loncası Caddesi“ heißt: siehe Kent Atlası, 232, B5. Es ist möglich, daß solche Keller als Versammlungsorte der Gewerbe- 
daß dieser Palastteil als Hochzeitsgeschenk errichtet worden war. Daraus ergibt sich, daß im Jahre 1146 einer der wichtigsten komnenischen Trakte des Palastes bereits vollendet war. ${ }^{535}$ Es ist daher recht wahrscheinlich, daß auch der Bau einer neuen Mauer in dieser früheren Periode von Manuel zumindest geplant, wenn nicht sogar bereits ausgeführt worden war.

Erstaunlicherweise wurde aber eine Passage von Niketas Choniates in diesem Zusammenhang nie berücksichtigt, die dafür einen wichtigen Beleg liefern könnte. Der byzantinische Historiker berichtet für das Jahr 1147 im Zusammenhang des Alamannengefahr - also ein Jahr nach der Hochzeit von Bertha und Manuel: $:^{336}$

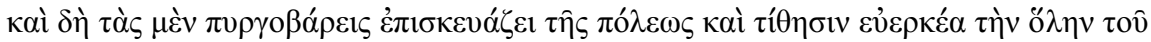

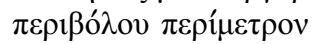

Der Kaiser ließ also die Befestigungsanlagen der Stadt in ihrem ganzen Umfang instand setzen und verteidigungsbereit machen

Odo von Deuil, der im selben Jahr in Konstantinopel weilte, berichtet über die Befestigungsmauer der Westseite der Stadt folgenderweise: ${ }^{537}$

Latus tertium de trigono civitatis campos habet, sed duplici muro munitur et turribis qui a mari usque ad Palatium fere duobus milibus tenditur. Hic nec robore firmus est nec turres in altum subrigit.,

The third side of the city's triangle include fields, but it is fortified by towers and a double wall which extends for about two miles from the sea to the palace. This wall is not very strong, and it possesses no lofty towers.,

Mit der von Odo von Deuil im zweiten Satz erwähnten Mauer, die nicht sehr stark gewesen sein soll und keine hohen Türme hatte, kann weder die im vorhergehenden Halbsatz beschriebene (theodosianische) Doppelmauer noch der Mauerabschnitt in den unteren Blachernen gemeint sein, da auch die drei Theophilos-Türme zu dieser Charakterisierung nicht passen würden. Demnach müßte es sich dabei entweder um die alte, wahrscheinlich von Herakleios errichtete Blachernen-Mauer oder um die Komnenen-Mauer gehandelt haben, die wahrscheinlich gegen 1147 gerade fertiggestellt worden war.

treibenden benutzt wurden und „Lonca“ dadurch eine übertragene Bedeutung gewann. Nach Restle, Istanbul, 296, stand der Palast möglicherweise oberhalb der Substruktionen. Eyice, Tekfur Sarayı, 233, äußerte, daß der Palastteil an der Stelle des Tekfur Sarayı gesucht werden könnte. Eyices Annahme basiert auf den an der Fassade befindlichen Lisenen, die er mit dem Torbau von Lorsch verglich; vgl. auch Wulzinger, Baudenkmäler, 87.

535 Magdalino, Manuel, 117. - Bis zum Jahre 1157 müssen bereits mehrere Teile der Palastanlage fertiggestellt gewesen sein; vgl. Magdalino, Constantinople, 69-70.

536 Niketas Choniates, 62/93-95; deutsche Übersetzung: Grabler, Krone der Komnenen, 96.

537 Odo of Deuil, IV, 65. 
Um die Frage beantworten zu können, welcher Teil der Mauer (Nord- oder Südtrakt) auf die erste Bauphase zurückgehen könnte, wäre auch ein Vergleich des Mauerwerks mit Bauten des Blachernen-Palastes notwendig. Obwohl von den Palasttrakten im Blachernen-Viertel nicht viel erhalten ist, sind einige Mauerfragmente in der Nähe der İvaz Efendi Camii südöstlich der Substruktionen bzw. nahe der Emir Buhari Tekkesi nordöstlich der Substruktionen (Abb. 34, 35), ${ }^{538}$ ferner weitere Terrassenmauerreste hinter der heutigen Blachernen-Kirche (Abb. 33, 34, 172, 173) ${ }^{539}$ und die hohen Blachernen-Substruktionen (sog. Anemas-Gefängnis, Abb. 33-35, 169, 171) ${ }^{540}$ noch vorhanden. Das Mauerwerk an der westlichen Straßenseite (vor der İvaz Efendi Camii) war fragmentarisch erhalten (im Januar 2007 mit einer modernen Überdeckung unsichtbar gemacht). Die wenigen an der östlichen Straßenseite unterhalb der Emir Buhari Tekkesi sichtbaren Fragmente zeigen verdeckte Schichttechnik. An der Terrassenmauer westlich der heutigen Blachernen-Kirche erkennt man ebenfalls verdeckte Schichttechnik mit vier sichtbaren Ziegellagen und Quadern mit Ziegeln in den Lagefugen. Vergleichbares Mauerwerk beobachtet man an den hohen Substruktionen (Abb. 169, 171), ${ }^{541}$ wobei dieser Teil 2006 intensive Restaurierungen erfuhr. Besonders die Ähnlichkeit zwischen dem Mauerwerk der Terrasse hinter der Blachernen-Kirche (Abb. 172, 173) und dem Nordtrakt der Komnenen-Mauer (Abb. 148, 152) ist augenscheinlich, doch können wir die Terrassenmauer keiner bestimmten komnenischen Epoche zuweisen.

Ein weiterer Mauerteil muß in diesem Zusammenhang erwähnt werden, und zwar der ca. $12 \mathrm{~m}$ breite „Vorsprung", der sich südlich von Turm 14, dem sog. Isaakios Angelos-Turm, befindet (Abb. 23, 35, 174). Auf den Fotos von Van Millingen und Meyer-Plath/Schneider sind die unteren Teile dieses aus kleinen Quadern ohne Ziegeln erbauten und mit Wandvorlagen verstärkten „Vorsprungs“, dessen Südwestteil noch zusätzlich verstärkt war, ${ }^{542}$ nicht zu erkennen, da er damals gänzlich von dichtem Buschwerk verdeckt war. ${ }^{543}$ Sein Mauerwerk konnte daher nicht untersucht werden. Der untere Bereich der Nordflanke des „Vorsprungs“ läßt sich wegen des angeschlossenen Sockels des

538 Dirimtekin, 14. Mintıka, 193-222.

539 Vgl. Anm. 534.

540 Siehe dazu Van Millingen, Walls, 131-163; Meyer-Plath/Schneider, Landmauer, 114 117 (Meyer-Plath).

541 Siehe dazu Meyer-Plath/Schneider, Landmauer, 114-117 (Meyer-Plath).

542 Es muß berücksichtigt werden, daß dieser Teil ca. zwei Meter unter dem heutigen Bodenniveau liegt und die oberen Teile später mit unregelmäßigen Quadern ohne Ziegeln renoviert wurden. Daher können wir nicht nachvollziehen, wie das gesamte ursprüngliche Mauerwerk aussah.

543 Meyer-Plath/Schneider, Landmauer, Taf. 57b; siehe auch Foss, Fortifications, 65, wo der Vorsprung als „a square projecting bastion“ bezeichnet wird. Auf einem Foto von Mehmet Ziya Bey, Boğaziçi, 153, sind die unteren Teile des Vorsprunges gut zu erkennen. 
Isaakios Angelos-Turms nicht überprüfen (Abb. 171). Die heute freigelegte Westseite und die schmale Südflanke zeigen noch das ursprüngliche Mauerwerk (Abb. 23, 174). Es besteht aus drei bzw. vier Quaderschichten mit Ziegeln in den Lagefugen und vierbändiger verdeckter Schichttechnik. Folgende Werte sind zu beobachten: Ziegelbandhöhe $75 \mathrm{~cm}$, Ziegelbreite $33 \mathrm{~cm}$, Ziegelhöhe $4 \mathrm{~cm}$, Mörtelhöhe 11 bis $12 \mathrm{~cm}$. Der „Vorsprung“ gehört demnach ebenfalls der komnenischen Zeit an. Obwohl die oben erwähnten Reste keinem bestimmten komnenischen Kaiser zugeschrieben werden können, ist die Ähnlichkeit des fast einheitlichen Mauerwerks mit dem am Nordtrakt der Komnenen-Mauer augenscheinlich.

Hier kann auf einen weiteren Mauerabschnitt aufmerksam gemacht werden: Das Mauerwerk um Tekfur Sarayı wurde bereits von Meyer-Plath/Schneider beschrieben, ${ }^{544}$ wobei besonders die unteren Teile an der Südfassade (Abb. 176) sowie die Kurtine hinter Vorturm 96a zum Zeitpunkt ihrer Arbeit teils unter dem Schutt lagen, teils durch Häuser verdeckt waren. ${ }^{545}$ In diesem Abschnitt der Mauer, besonders in den wieder sichtbaren unteren Partien sind Reste aus drei bzw. vier Ziegelreihen bestehender Ziegelbänder in verdeckter Schichttechnik zu erkennen (Abb. 27a, 179), das auch Foss registrierte und als „quite similar to the north section of the walls of Manuel Comnenos" bezeichnete. ${ }^{546}$ Die Verwandtschaft mit dem "Vorsprung" (Abb. 174) ist ebenfalls augenfällig und zeigt, daß im Bereich von Tekfur Sarayı komnenische Bautätigkeit stattfand. Daraus ergibt sich ein weitere Indiz, wonach die aus dem gleichen Bauprogramm stammende Komnenen-Mauer ursprünglich nicht wie der Südtrakt, sondern wie der Nordtrakt (Abb. 148) ausgesehen haben wird. Erst in der zweiten Bauphase muß die Südpartie von Turm 9 bis zum Tekfur Sarayı durch die gewaltigen Türme ersetzt und bewußt mit den mächtigen Quadern errichtet worden sein. ${ }^{547}$

Während am Nordtrakt der Mauer, abgesehen von drei Büsten (Abb. 158, 159a, b, c), die oberhalb des Tors an der Kurtine 11/12 angebracht sind (siehe Abschnitt 3.4.3.), keinerlei skulptierte Spolien festgestellt werden können, befinden sich im Südtrakt mehrere solcher Reste, die nicht zur Dekoration verwendet worden sind. Sie ergänzen hier in der Regel gewöhnliches Baumaterial, wobei sie, ohne auf die ornamentalen Motive zu achten, zum Teil umgekehrt eingemauert sind (Abb. 194). Eine derartige Verwendung skulptierter Spolien begegnet auch an anderen Abschnitten der Stadtmauern (siehe Abschnitt 4.2.),

544 Meyer-Plath/Schneider, Landmauer, 105-108 (Meyer-Plath).

545 Meyer-Plath/Schneider, Landmauer, Taf. 44a, 47a/b.

546 Foss, Fortifications, 64.

547 Die verdeckte Schichttechnik kommt zwar auch in palaiologischer Zeit vor, doch nur noch gelegentlich; siehe Hallensleben, Pammakaristos, 166; für weitere Literatur zur verdeckten Schichttechnik vgl. Anm. 525. 
die sehr rasch repariert worden sind, wie z. B. an Turm 83 der Theodosianischen Landmauer (Abb. 195, 196). Das spricht dafür, daß auch der Südtrakt in relativ kurzer Zeit neu aufgeführt wurde.

Somit stellt sich die Frage, wann und weshalb der Südtrakt der KomnenenMauer wiederhergestellt werden mußte. Dafür käme eigentlich nur ein Erdbeben als Ursache in Betracht. In der Sekundärliteratur ist jedoch von einem Erdbeben in dieser Zeit keine Rede. Den einschlägigen Erdbebenlisten zufolge haben sich in der Hauptstadt zwischen 1090 und 1202 - also über hundert Jahre - überhaupt keine Erdbeben ereignet. ${ }^{548}$ Doch gab es in Konstantinopel innerhalb dieses Zeitraums eine große Erdbebenkatastrophe, die in der bisherigen Forschung keine Beachtung gefunden hat. Niketas Choniates berichtet im Zusammenhang mit dem Staatsbesuch des Seldschukensultans Kılıç Arslan II. (1156-1188) im Jahre 1162: ${ }^{59}$

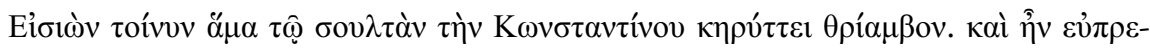

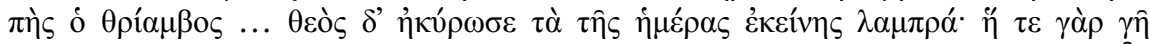

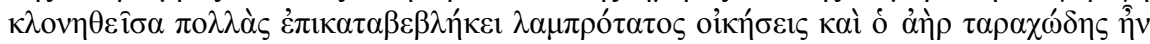

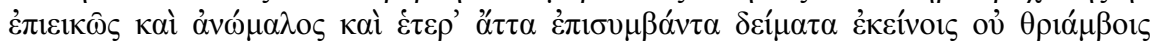

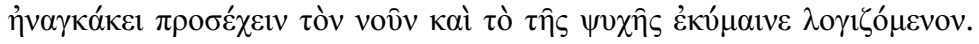

Er [Manuel] wollte mit dem Sultan in Konstantinopel einziehen und ordnete einen Triumphzug an. Dieser Zug war großartig (geplant) ... Aber Gott machte den Glanz und die Pracht dieses Tages zunichte: Die Erde bebte und ließ viele herrliche Bauwerke einstürzen, und ein ungewöhnlich heftiger Sturm wütete. Das und all der andere dadurch verursachte Schrecken zwang die Menschen, an ihr eigenes Leben zu denken, und keiner achtete mehr des Triumphzuges.

Inwieweit dieses Erdbeben Schäden an den Stadtmauern und vor allem der Komnenen-Mauer angerichtet hatte, entzieht sich unserer Kenntnis, zumal Niketas Choniates nur von zerstörten Bauten der Stadt redet. Ein Indiz zeigt aber eindeutig, daß auch die Stadtmauern von dieser Katastrophe betroffen waren und zumindest die Marmara-Seemauer gründlich repariert werden mußte. Turm 92 der Marmara-Seemauer ${ }^{50}$ im Bereich des heutigen Stadtviertels Narlıkapı trägt nämlich eine Reparaturinschrift des Kaisers Manuel, die nicht nur seinen Namen, sondern auch die Jahreszahl 1163/64 wiedergibt. ${ }^{551}$ Der Bericht des Niketas Choniates und die Turminschrift lassen annehmen, daß

548 Downey, Earthquakes, 600; Grumel, Chronologie, 480.

549 Niketas Choniates, 118/38-119/47; deutsche Übersetzung: Grabler, Krone der Komnenen, 158. - Auch Ioannes Kinnamos, 206/19-23, erwähnt dieses Erdbeben.

550 Bei der Numerierung wurde Dirimtekin, Marmara, zugrundegelegt.

551 Das Inschriftband ist heute nur noch teilweise erhalten; vollständig wiedergegeben bei Mordtmann, Esquisse, 60, und Van Millingen, Walls, 187; siehe auch Dirimtekin, Marmara, 49, Abb. 115 (ganzer Turm), Abb. 103 (Detailaufnahme der Inschrift); Mango, Inscriptions, 55. - Schneider, Yedikule, 198, erwähnt ebenfalls die Inschrift und nimmt an, daß die Mauern im Verlauf der Zeit naturgemäß repariert werden mußten. 
durch dieses Erdbeben mit großer Wahrscheinlichkeit auch die KomnenenMauer und vor allem der an einem steilen Abhang stehende Südtrakt beschädigt worden waren, weswegen dieser in einer abweichenden Technik und mit anderem Material, aber noch stattlicher und stärker wiederhergestellt wurde.

Nach den Schriftquellen und dem Befund können die Bauphasen der Komnenen-Mauer folgenderweise aufgelistet werden:

1146/47: Mit Mauerwerk B ausgeführte Türme und Kurtinen zwischen Tekfur Sarayı-Nordturm bis Turm 12 bzw. etwas nördlich davon (siehe Abschnitt 3.3.). Diese Mauer war wahrscheinlich die von Odo de Deuil registrierte, die ihm zu Recht als nicht sehr stark vorkam. Innerhalb des gleichen Bauprogramms müssen auch mehrere Trakte des Blachernen-Palastes errichtet worden sein.

1162: Erdbeben und Zerstörung erheblicher Teile der Mauer.

Nach 1162 (wahrscheinlich 1163/64): Wiederherstellung des Südtrakts, wobei in den jetzt im Mauerwerk A neu errichteten Teilen auch mehrere Spolien als Ersatz für Baumaterial wiederverwendet worden sind.

\subsection{Palaiologische Reparaturen der Komnenen-Mauer}

Die Komnenen-Mauer weist aber weitere Reparaturen auf, wobei die oben erwähnten Mauertechniken C (Abb. 20, 21, 155) und D (Abb. 160, 162) verwendet worden sind. Das Mauerwerk D kommt überwiegend an den oberen Teilen der Kurtinen und Türme vor, wobei Kurtine 12/13 gänzlich in diesem wiederhergestellt wurde (Abb. 160). Vergleichbares Mauerwerk begegnet auch an der Theodosianischen Landmauer besonders im Bereich der Vormauer. Sowohl die an Kurtine 12/13 registrierte Inschrift, die den Namen Ioannes' VIII. Palaiologos und das Baudatum 1441 erwähnt, ${ }^{552}$ als auch weitere Inschriften an der Vormauer der Theodosianischen Landmauer, die ebenfalls auf die gleiche Periode hinweisen (Abb. 53, 59, 60), ${ }^{553}$ lassen keinen Zweifel daran, daß es sich bei Mauerwerk D um spätbyzantinische Reparaturen womöglich des 15. Jahrhunderts handelt. ${ }^{554}$ Diese fast ohne Ziegeln ausgeführten Teile unterscheiden sich von dem mit mehreren dünnen Ziegellagen hergestellten Mauerwerk $(C)^{555}$ und legen die Annahme nahe, daß zwischen diesen beiden Mauerwerksarten eine gewisse Zeitspanne liegen könnte (Abb. 20, 155).

552 Vgl. Meyer-Plath/Schneider, Landmauer, 140, Nr. 59 (Schneider); Foss, Fortifications, 51.

553 Für weitere Beispiele siehe Meyer-Plath/Schneider, Landmauer, 127, Nr.11, Nr. 17 (Schneider).

554 Meyer-Plath/Schneider, Landmauer, 26 (Meyer-Plath); vgl. Foss, Fortifications, 59.

555 Vgl. Anm. 527. 
Die Schriftquellen setzen die frühpalaiologischen Reparaturen der Stadtmauern von Konstantinopel in die Regierungszeit Michaels VIII. Palaiologos (1259-1282), wonach sich dieser Kaiser jedoch hauptsächlich auf die Reparatur der Marmara-Seemauer konzentriert haben soll. ${ }^{556}$ Es ist aber sehr wahrscheinlich, daß nach fast sechzigjähriger Lateinerherrschaft auch die Mauerlinie zwischen Marmarameer und Goldenem Horn baufällig war und ebenfalls repariert werden mußte. ${ }^{557}$ Die Restaurierung der Stadt und die Reparaturtätigkeit an den Stadtmauern wurden den Quellen zufolge unter Andronikos II. Palaiologos $(1282-1328)$ fortgeführt. ${ }^{558}$

Wie oben erwähnt, kommt Mauerwerk $\mathrm{C}$ an den stadtseitigen Kurtinen 9/ 10 (Abb. 21) und an der stadtseitigen Wehrgangstreppe hinter Turm 8 vor (Abb. 155). Auch die Kurtine Tekfur Sarayı-Nordturm/Turm 1 weist vergleichbares Mauerwerk auf (Abb. 20), und zwar bis auf die beiden Turm 1 nördlich benachbarten stadtseitigen Nischen. Obwohl hier keine Baunaht zu erkennen ist, sind nicht nur die abrupte Änderung des Mauerwerks augenfällig, sondern auch die unterschiedlichen Breiten der Nischen. ${ }^{559}$ Während die beiden dem Turm benachbarten Nischen eine Breite von 2,30 m aufweisen, beträgt diese an den übrigen Nischen, die mit Mauerwerk $\mathrm{C}$ ausgeführt sind, 1,50 m.

In keinem dieser Bereiche ist heute irgendeine Reparaturinschrift in situ zu finden, welche die Bautätigkeit der Kaiser der frühpalaiologischen Epoche bestätigen und für unsere Fragestellung von Bedeutung sein könnte. Doch muß es noch im 16. Jahrhundert an der Komnenen-Mauer eine Inschrift aus dieser Periode gegeben haben: Sowohl das Wiener ${ }^{560}$ als auch das Cambridger ${ }^{561}$ Torverzeichnis - beide von Ioannes Malaxos angefertigt - enthalten eine Liste der Tore an den Stadtmauern von Konstantinopel, in denen Eğrikapı zwischen den Türmen 6 und 7 der Komnenen-Mauer als fünfzehntes Tor der Landmauer bezeichnet wird (Abb. 156, 157). Beide Torverzeichnisse berichten von einer

556 Quellen und Diskussion bei Talbot, Restoration, 243-261; Kidonopoulos, Bauten, $232-242$.

557 Für den Zustand der Stadt um 1261 vgl. vorige Anm.

558 Siehe Nikephoros Gregoras, 470/2-3; deutsch: Van Dieten, Nikephoros, II, 2, 244. Eine Platte mit Wappen und Monogramm wurde in der Marmara-Seemauer in der Nähe von Kumkapı von Curtis/Walker, Restes, Nr. 1, registriert und von Van Millingen, Walls, 189, mit Andronikos II. Palaiologos in Verbindung gebracht. Mango, Constantinopolitana, 335, Anm. 91, äußerte Zweifel, ob sich das Wappen auf Andronikos II. beziehen läßt. Von diesem Teil der Seemauer sind heute fast keine Reste erhalten; vgl. Dirimtekin, Marmara, 34.

559 Vgl. Anm. 520.

560 Wien, Österreichische Nationalbibliothek, Cod. Hist. gr. 94 , fols. $29^{\mathrm{r}}-35^{\mathrm{r}}$; siehe dazu Preger/Pantchenko, Verzeichnis, S.461-471; Mango, Inscriptions, 54, Nr. 9.

561 Cambridge, Trinity College, Cod. O. 2.36, fols. $162^{\mathrm{r}}-164^{\mathrm{v}}$; siehe dazu Schreiner, Pammakaristos, 217-248; zu Ioannes Malaxos siehe De Gregorio, Malaxos, 189-241, 258-259; Schreiner, Malaxos, 203-214. 
Inschrift, die sich etwas oberhalb dieses Tors, also in Richtung Süden befunden haben soll. Die Notiz im Wiener Torverzeichnis lautet: ${ }^{562}$

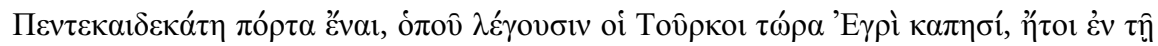

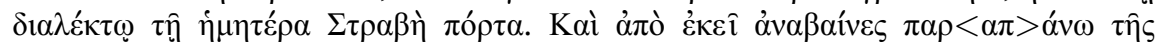

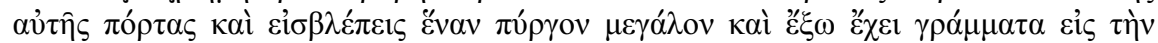

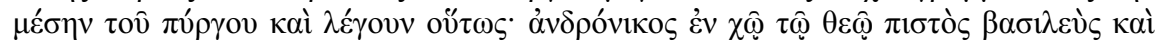

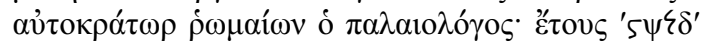

Das fünfzehnte Tor ist das, welches die Türken als Eğri Kapı bezeichnen, was in unserer [griechischen] Sprache Schiefes Tor bedeutet. Wenn man von dort etwas oberhalb von diesem Tor hinaufgeht, erblickt man einen großen Turm und außen ist eine Inschrift in der Mitte des Turms, die folgendermaßen zu lesen ist: Andronikos Palaiologos, Christus ergebener Basileus und Autokrator der Rhomäer. Jahr $1285 / 86$

Die Torverzeichnisse erwähnen noch zwei weitere Inschriften, die etwas weiter südlich angebracht waren. Die eine soll den Namen des Kaisers Ioannes V. Palaiologos und das Datum 1362/63 wiedergegeben haben. ${ }^{563}$ Die andere befand sich im Bereich von Tekfur Sarayı, worauf der Name des Kaisers Manuel II. Palaiologos mit der Jahreszahl 1384/85 zu lesen gewesen sei. ${ }^{564}$ Nach diesen drei Inschriften wird in beiden Verzeichnissen als sechzehntes Tor Edirnekapı unter seinem damals üblichen Namen Adrianopolis-Tor angegeben. Meyer-Plath/ Schneider zufolge beziehen sich alle drei Inschriften im Wiener Torverzeichnis (das Cambridger war noch unbekannt) auf die Theodosianische Landmauer. Sie begründeten ihre Meinung damit, daß die Komnenen-Mauer kaum Reparaturen aufweise. ${ }^{565}$ Dieser Aussage widerspricht aber eine andere Stelle des gleichen Buchs, wo auf mehrere Reparaturen an dieser Mauer hingewiesen wird. ${ }^{566}$

Da die dritte Inschrift mit dem Namen Manuels II. Palaiologos im Zusammenhang mit Tekfur Sarayı, das direkt hinter dem südlichen Anschluß an die Komnenen-Mauer steht, erwähnt wird, kann die im Wiener sowie im Cambridger Torverzeichnis erwähnte Inschrift mit dem Namen Andronikos' II. Palaiologos sich m.E. nicht an der Theodosianischen Landmauer befunden haben, sondern nur zwischen Eğrikapı und Tekfur Sarayı. Im Wiener Torverzeichnis ist von einem großen Turm die Rede, woran sich die Inschrift des

562 Preger, Tore, 470, Nr. 15. - Im Cambridger Torverzeichnis wird die Größe des Turms nicht hervorgehoben; siehe Schreiner, Pammakaristos 243, Nr. 15.

563 Preger/Pantchenko, Verzeichnis, 470, unter Nr.15; Schreiner, Pammakaristos, 243, unter Nr. 15.

564 Preger/Pantchenko, Verzeichnis, 470, unter Nr. 15; Schreiner, Pammakaristos, 243, unter Nr. 15. - Bereits Schneider (Meyer-Plath/Schneider, Landmauern, 144 zu Nr. 82) hatte erkannt, daß die Jahreszahl nicht zutreffen kann, da Manuel erst 1391 auf den Kaiserthron kam.

565 Meyer-Plath/Schneider, Landmauer, 144 (Schneider).

566 Meyer-Plath/Schneider, Landmauer, 111-114 (Meyer-Plath). 
Kaisers Andronikos II. Palaiologos befand. Meyer-Plath/Schneider wollten diesen Turm mit der Theodosianischen Landmauer in Verbindungen bringen und plazierten die Inschrift an dem großen Vorturm, also an Turm 96a (Abb. 175). ${ }^{567}$ Sowohl auf Grund der topographischen als auch der architektonischen Hinweise kann der erwähnte „große Turm“ nur Eckturm 3 der Komnenen-Mauer gewesen sein, der mit seinen Dimensionen alle anderen Türme dieses Bereichs übertrifft (Abb. 33, 34, 147). Der Turm steht darüber hinaus an einer Stelle, wo in beiden Richtungen Reparaturen in der Mauertechnik C zu beobachten sind.

Wir wissen nur nicht, ob diese Arbeiten unmittelbar nach einer Naturkatastrophe stattfanden, oder ob es dabei um einer Fortsetzung der von Michael VIII. Palaiologos begonnenen Maßnahmen handelte. Es scheint aber, daß die Reparaturtätigkeiten Andronikos' II. Palaiologos sich nicht auf das Jahr 1285/86 beschränkten. Ein am stadtseitigen Teil der Kurtine 12/13 (Abb. 162), die 1441 errichtet bzw. repariert wurde, umgekehrt eingemauertes Inschriftfragment muß im Zusammenhang mit den frühpalaiologischen Reparaturen dieser Mauer ebenfalls berücksichtigt werden. Der Inschriftrest erwähnt das Datum 1317 bzw. 1318, gehört somit der Regierungszeit dieses Kaisers an. ${ }^{568}$ Die auf einem schmalen Band angebrachten Buchstaben lassen keinen Zweifel daran, daß es sich dabei um eine wiederverwendete Reparaturinschrift der Mauer handelt. Das angegebene Datum markiert das Todesjahr der Kaiserin Eirene, die ihrem Mann eine erhebliche Menge Geldes hinterlassen hatte. ${ }^{569}$ Die Inschrift stammt entweder aus der Vorgänger-Kurtine oder aus einer anderen von diesem Kaiser reparierten Stelle der Blachernen-Mauer. Sie datiert ein Jahr nach der umfassenden Reparatur der Hagia Sophia im Jahre $1317 .{ }^{570}$ Es wurde bisher nicht endgültig geklärt, weshalb die Hauptkirche und die Stadtmauern um diese Zeit repariert werden mußten, und ob es sich auch dabei um ein sonst unbekanntes Erdbeben gehandelt haben könnte. In den Erdbebenlisten von Downey ${ }^{571}$ und Wirth ${ }^{572}$ wird keine solche Katastrophe zwischen 1296-1323 erwähnt. Dagegen spricht die Kleinchronik Nr. 9 von einem großen Erdbeben, das am

567 Meyer-Plath/Schneider, Landmauer, 6, Anm. 1, 144, Nr. 84 (Schneider), als Turm 97 numeriert.

568 Paspatis, Meletai, 41, las das Datum der Inschrift als 1317, so auch Van Millingen, Walls, 126; dagegen Meyer-Plath/Schneider, Landmauer, 140, Nr. 58, zum Jahr 1318 (Schneider).

569 Nikephoros Gregoras, 273/14-25; deutsch: Van Dieten, Nikephoros, I, 206; vgl. auch Van Millingen, Walls 103 und 126.

570 Mainstone, Hagia Sophia, 102-103. - Zur umfangreichen Bautätigkeit in Konstantinopel unter Kaiser Andronikos II. Palaiologos siehe Anm. 556; vgl. Ousterhout, Developments, 75-91.

571 Downey, Erdbeben, 600.

572 Wirth, Erdbebenliste, 393-399. 
29. September 1315 stattfand. ${ }^{573}$ Dieses Erdbeben muß nicht nur an der Hagia Sophia, sondern auch an Teilen der Mauern Schäden verursacht haben, weswegen hier Reparaturen notwendig wurden. ${ }^{574}$ Sowohl die erhaltene Inschrift als auch das Todesjahr der Kaiserin sowie die Reparatur der Hagia Sophia können mit der Nachricht über das Erdbeben von 1315 in Verbindung gebracht werden. Es ist daher sehr wahrscheinlich, daß bald nach diesem Erdbeben ebenfalls mehrere Stellen der Blachernen- bzw. Komnenen-Mauer instand gesetzt werden mußten. Dadurch wird der Grund für die zweite Reparaturtätigkeit des Kaisers faßbar sowie bestätigt, daß das Mauerwerk $\mathrm{C}$ aus der früheren Regierungszeit Andronikos' II. Palaiologos stammt.

\subsection{Der nördlichste Abschnitt der Komnenen-Mauer, Kurtine 13/14 und Turm 13}

Die Komnenen-Mauer hört unmittelbar nach Turm 12 auf (Abb. 33, 34, 148, vorletzter Turm vor den Substruktionen). Es beginnt dort die spätere Kurtine 12/13, die inschriftlich 1441 datiert wird (Abb. 160, 162). Wie soeben dargelegt, war sie möglicherweise schon einmal in palaiologischer Zeit, und zwar im Jahre 1317/18 repariert worden. Sie trifft an der Südostecke von Turm 13 mit Kurtine 13/14 zusammen - gemeint ist der Abschnitt bis zu dem schon mehrmals erwähnten „Vorsprung“ (Abb. 23, 174). Kurtine 13/14 wurde mit einem cloissonnéartigen Mauerwerk errichtet (Abb. 23), das unterschiedlich datiert wird. ${ }^{575}$ Bislang blieb unklar, ob Kurtine 12/13 einen komnenischen Vorgänger hatte, oder ob die Komnenen-Mauer tatsächlich an der Nordseite von Turm 12 endete.

Zunächst soll die Eckverbindung zwischen „Vorsprung“ und dem cloissonnéartigen Mauerwerk näher in Augenschein genommen werden. Das Cloissonnémauerwerk verbindet sich mit dem in die Zeit Manuels I. Komnenos datierbaren nördlichen „Vorsprung" nicht organisch und scheint früher als dieser entstanden zu sein (Abb. 23). Trotzdem läßt die verdeckte Schichttechnik diese Mauer in die komnenische Zeit datieren, obwohl zum Teil dickere und möglicherweise wiederverwendete Ziegel zu beobachten sind (Abb. 26). Das cloissonnéartige Mauerwerk verläuft hinter Turm 13 weiter bis zu dessen

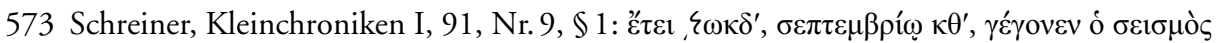

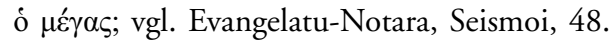

574 Nikephoros Gregoras, I, 273/12, erwähnt das Erdbeben nicht.

575 Meyer-Plath/Schneider, Landmauer, 25 (Meyer-Plath) haben diesen Abschnitt als Kurtine 11/12 angegeben; ebd. 114 (Meyer-Plath) datierten sie zunächst die oberen Partien, die mit unregelmäßigen Quadern repariert wurden, in palaiologische Zeit; ebd.,21, Taf. 21a, wird das Mauerwerk im ganzen palaiologisch datiert. Foss, Fortifications, 76, schlug eine Datierung ca. 1170 vor. 
Südflanke (Abb. 164) und bricht hier stadteinwärts um, wobei die Fortsetzung im Garten eines Privatgrundstücks noch zu erkennen ist (Abb. 162, 163). Eine Fortsetzung wurde zwar auch von Van Millingen gezeichnet, doch verband der Autor sie mit der Mumhane-Mauer (Abb. 34, letzter Turm vor den Substruktionen). ${ }^{576}$ Zur Zeit von Meyer-Plath/Schneider war dieser Bereich bereits verschüttet. ${ }^{577} \mathrm{Ihr}$ Verlauf macht deutlich, daß es sich bei dem cloissonnéartigen Mauerwerk nicht um ein willkürlich eingefügtes Zwischenmauerstück, sondern um die Grenze bzw. die Substruktion eines bestimmten Teils des BlachernenPalastes gehandelt haben wird, worauf heute moderne Häuser stehen, deren Bewohner die byzantinischen Substruktionen als Keller nutzen. ${ }^{578}$ Damit wird noch einmal bestätigt, daß das cloissonnéartige Mauerwerk früher als die Mauer Manuels I. Komnenos errichtet wurde und die von ihm die zum Schutz des Blachernen-Palastes errichtete Mauer ursprünglich bis zu dem erwähnten Knick reichte. Wie es sich jedoch mit den großen Substruktionen an der Nordseite verbindet, ist nicht mehr nachzuvollziehen.

Vor dem südlichen Teil des cloissonnéartigen Mauerstücks wurde Turm 13 errichtet (Abb. 164, 166), dessen Südostecke eine zusätzliche Verstärkung erfuhr. Er unterscheidet sich in mehrerlei Hinsicht von den benachbarten Türmen 11 und 12 der Komnenen-Mauer (Abb. 148). Der Turm enthält an der Westseite eine Inschrift, die den Namen des Kaisers Isaakios II. Angelos und das Datum 1186/87 wiedergibt (Abb. 167) ${ }^{579}$ Es wird zumeist angenommen, daß es sich dabei um eine Spolie handelt und die Inschrift ursprünglich zu Turm 14 gehört habe. ${ }^{580}$ Auch wird die Meinung vertreten, daß die Inschrift schon ursprünglich hier angebracht war und Turm 13 daher ebenfalls dem Ensemble des Kaisers Isaakios Angelos zurechnet werden könnte (Abb. 170). ${ }^{581}$ Der Turm weist ein unordentliches Mauerwerk auf. Die fünfschichtig angeordneten und sehr unregelmäßigen Quader haben zwei bis drei Ziegelschichten in den Lagefugen. Das Ziegelband ist verhältnismäßig schmal im Vergleich zu den Quaderlagen. Die Ziegel und die Quader scheinen mehrmals verwendet worden zu sein (Mauerprobe: Ziegelbandhöhe ca. $38 \mathrm{~cm}$, Ziegelbreite 15 bis $30 \mathrm{~cm}$,

576 Vgl. auch Tsangadas, Fortifications, Taf. III.

577 Meyer-Plath/Schneider, Landmauer, Taf. 40.

578 Für die über dieser Mauer befindliche hohe Plattform mit Gebäuden westlich der İvaz Efendi Camii siehe die Abb. bei Van Millingen, Walls, gegenüber 166, und MüllerWiener, Topographie, 507, Abb. 351.

579 Van Millingen, Walls, 132, las das Datum als 1188. Meyer-Plath/Schneider, Landmauer, 140, Nr. 60, hatten das Datum als 1186/87 entziffert; ebd., 102, wurde der Turm in das Jahr 1188 datiert (Schneider).

580 Paspatis, Meletai, 39, behauptete, daß die Inschrift ursprünglich am benachbarten Turm 14 angebracht war; zustimmend Meyer-Plath/Schneider, Landmauer, 102 (Schneider).

581 Wie z. B. Foss, Fortifications, 58: „It seems probable that tower B. 13, which had a large window inappropriate for defence on its north side, and B. 14 formed part of the same project of expansion of the palace of Blachernae." - Vgl. Anm. 586. 
Ziegelhöhe 2,5 bis $5 \mathrm{~cm}$, Mörtelhöhe $3 \mathrm{~cm}$ ). Zum Teil kommen auch vertikal eingefügte Ziegel vor (Abb. 167). Das Mauerwerk weicht also von dem bereits besprochenen Mauerwerk des Nordtrakts der Komnenen-Mauer (Abb. 149) $a b{ }^{582}$ Der Unterschied zu dem Andronikos II. zugeschriebenen Mauerwerk C (Abb. 20) ist ebenfalls augenfällig. Die Bauweise von Turm 13 (Abb. 166) kann auch nicht dem aus reinen Quadern erbauten spätpaläologischen Mauerwerk zeitlich gleichgesetzt werden (Abb. 160, 162).

Der Turm war zur Zeit von Van Millingen und auch noch während der Arbeiten von Meyer-Plath/Schneider wegen der hohen Verschüttung unzugänglich. Meyer-Plath/Schneider beschrieben ihn folgendermaßen: ${ }^{583}$

Vierseitig, nur die unteren zwei Drittel erhalten, Inneres verschüttet ... An der Nordflanke in Obergeschoßhöhe eine türähnliche Öffnung, darunter Turmpforte, entsprechend auch an der Südflanke, 2,15 m von der Kurtine entfernt.

Da seine Nordflanke abgestürzt ist, ist der Turm heute zugänglich. Die heutige Nordwand war ursprünglich eine Zwischenwand, welche die untere Etage des Turms in zwei Räumlichkeiten teilte (Abb. 168). Die in der Zwischenwand befindliche Tür war demzufolge ein Durchgang zwischen diesen beiden Räumen. Auf einem alten Foto bei Van Millingen ist zu erkennen, daß die zerstörte Nordflanke die eigentliche Pforte enthielt, die in den nördlichen Raum führte. ${ }^{584}$ Vom nördlichen Raum ist nur ein aus der Westseite ca. $3 \mathrm{~m}$ nach Norden herausragender Mauerrest erhalten. Ein weiterer Durchgang, von dem heute wegen der hohen Verschüttung nur der obere Bogen zu sehen ist, führte von außen in die südliche Kammer, die einen trapezoiden Grundriß aufweist (Rückwand: 2,70 cm, Westwand 3,20 cm, Flanken: ca. $8 \mathrm{~m}$ ). Die obere Etage muß ebenfalls aus zwei Räumlichkeiten bestanden haben, da die Zwischenwand bzw. die Tür auch hier vorhanden ist. Auf dem erwähnten Foto von Van Millingen ist weiterhin ein Fenster in der oberen Etage der Nordflanke zu sehen. Ob die Südflanke ein solches besaß, kann wegen der späteren Reparaturen nicht mehr festgestellt werden.

Auch aufgrund dieser architektonischen Besonderheiten unterscheidet sich Turm 13 von allen anderen, da er nicht wie ein gewöhnlicher Wehrturm aussieht. ${ }^{585}$ Er steht heute ungefähr zwei Meter in der Aufschüttung, weshalb der gesamte untere Abschnitt nicht überprüft werden kann. Obwohl sich endgültige Klarheit nur durch eine Sondage gewinnen ließe, scheint es sich um einen frühpalaiologischen Turm zu handeln. Seine Funktion und die Frage, ob hier

582 Foss, Fortifications, 58, verglich das Mauerwerk von Turm 13 mit dem Nordtrakt der Komnenen-Mauer. Meyer-Plath/Schneider, Landmauer, 114 (Meyer-Plath), halten es für palaiologisch.

583 Meyer-Plath/Schneider, Landmauer, 114 (Meyer-Plath).

584 Siehe Van Millingen, Walls, gegenüber 126.

585 Vgl. Van Millingen, Walls, 144. 
ein mittelbyzantinischer Vorgängerturm existierte bzw. welchen Bezug dieser zum Isaakios Angelos-Turm gehabt haben könnte, müssen vorläufig offen bleiben. ${ }^{586}$

\subsection{Zur Frage der Tore}

3.4.1. Die letzte Kaiserresidenz von Byzanz und das Palast-Tor

Wie bei den Toren der Landmauer bereitet auch die Identifikation der Tore der Blachernen-Mauer bzw. der Komnenen-Mauer Probleme. Die Quellen des 15. Jahrhunderts, besonders diejenigen, die über die Eroberung von Konstantinopel im Jahre 1453 berichten, erwähnen u. a. öfter eine Xyloporta ${ }^{587}$ und ein PalastTor als topographische Fixpunkte. Während die Xyloporta mit großer Übereinstimmung in der Ufersperrmauer zwischen Leon-Vorwerk und Goldenem Horn plaziert wird, ${ }^{588}$ geht die Lage des Palast-Tors aus den Quellen nicht eindeutig hervor. ${ }^{589}$ Abgesehen davon findet man nur im Bericht von Ioannes Kantakuzenos eine „Porphyrogennetos-Tor" bzw. „Porphyrogennetos-Pforte“ genannte Toranlage, ${ }^{590}$ die seit dem 19. Jahrhundert am Tekfur Saray1 lokalisiert wird. ${ }^{591}$ Zweifelsfrei gab es auch am Goldenen Horn Tore, die zum BlachernenPalast führten und von denen eines als Palast-Tor bezeichnet wurde. ${ }^{592}$ Doch wissen wir nicht, ob eines der Tore am Nordtrakt der Blachernen-Mauer - also der Komnenen-Mauer - ebenfalls Palast-Tor hieß und unter „Palast“ besonders im 15. Jahrhundert ein anderes Gebäude als der Palastkomplex im unteren Blachernen-Viertel verstanden wurde. Da wichtige historische Ereignisse und verschiedene Tornamen besonders in den Quellen des 15. Jahrhunderts des öfteren im Zusammenhang mit dem Kaiserpalast vorkommen, ist eine Vor-

586 Van Millingen, Walls, 145, äußerte: „But, on the hypothesis that L [Turm 13] and N [Turm 14] were both erected by Isaac Angelus, it is extremely strange that the inscription in his honour should have been placed upon the inferior tower, and not upon the one which formed his residence and had some architectural pretensions. This objection can be met, indeed, either by assuming that another inscription in honour of Isaac Angelus stood on the Tower N, but has disappeared; or, with Dr. Paspates, it may be maintained that the inscription is not in its proper place, but belonged originally to the counter-fort supportimg the tower $\mathrm{N}$, and was transferred thence to the tower $\mathrm{L}$ when the latter was repaired."

587 Vgl. Anm. 475.

588 Siehe vorige Anm.

589 Siehe Anm. 592.

590 Kantakuzenos, II, 138/6-7.

591 Van Millingen, Walls, 112.

592 Siehe zuletzt Effenberger, Buondelmonti, Tabelle III, Nr.3B. 
stellung davon, wo die letzten byzantinischen Kaiser residiert haben, für die topographische Forschung von Bedeutung.

Nördlich der Theodosianischen Landmauern sind uns zwei Palastanlagen bekannt: Der Blachernen-Palast in den unteren Blachernen, dessen Reste - die hohen Substruktionen (sog. Anemas-Gefängnis, Abb. 35, 169, 171) und die oben erwähnten Mauerfragmente bei der İvaz Efendi Camii bzw. bei der Emir Buhari Tekkesi sowie die Fragmente hinter der Blachernen-Kirche (Abb. 172, 173) - im heutigen Stadtviertel Ayvansaray noch zu erkennen sind, sowie Tekfur Sarayı (Abb. 6, 33, 175-178, 182, 183). Auf den komnenischen BlachernenPalastes wurde bereits oben kurz eingegangen. ${ }^{59}$ Während der Lateinerherrschaft wurde dieser Palast zum Teil von den lateinischen Kaisern als Residenz benutzt. In palaiologischer Zeit mußte er gründlich renoviert werden. ${ }^{594}$

Tekfur Sarayı besteht aus folgenden Bauteilen: ${ }^{595}$ 1.) Einem über der westlichen Kurtine nördlich des halbrunden Turms errichteten Längsbau (Abb. 178), 2.) der an der Innenseite der westlichen Kurtine angebrachten Rampe, die zum ungedeckten Wehrgang und von dort zum ersten Obergeschoß des Hauptgebäudes hinaufführte, mit dem genannten Turm und einer Toranlage (Abb. 182, 183), 3.) dem am Mauerknick quer in den Hof eingestellten Hauptgebäude (Abb. 6), dem der viereckige Turm 96a im Südwesten vorgelagert ist (Abb. 175), 4.) dem Hof mit der östlichen Hofmauer. Die untere Partie der Kurtine mitsamt dem halbrunden Turm und der nördlich davon gelegenen Pforte steckten lange in der neuzeitlichen Aufschüttung, ${ }^{596}$ doch im Sommer 2005 war die Stelle vor der Pforte (Feldseite) in der gesamten Höhe und Breite für eine kurze Zeit freigelegt worden (Abb. 181). Die nachträglich zugemauerte Pforte ist ca. 3,30 m (bis zum Sturz) hoch und hat eine lichte Weite von 3,55 m. ${ }^{597}$ Die sichtbaren Anschlüsse der Kurtine an die Pforte sind gänzlich aus den gleichen Quadern errichtet, deren oberste Reihe schon immer aus dem Schutt der Hofseite (Abb. 182, 183) sowie an der Feldseite herausragte (Abb. 181). Auch die Ostfassade des Palastes muß im ursprünglichen Zustand stärker akzentuiert gewesen sein. Der von Van Millingen an dieser Seite angedeutete Balkon ${ }^{598}$ ist auf einem Foto von Sébah und Joaillier (Ende 19./Anfang 20. Jh.) noch deutlich zu erkennen. ${ }^{599}$

593 Für die Nutzung des Palastes in palaiologischer Zeit siehe Tinnefeld, Blachernenpalast, $277-285$.

594 Überblick über die Quellen bei Müller-Wiener, Topographie, 224; Kidonopoulos, Bauten, 150-151; Tinnefeld, Blachernenpalast, 277-285; vgl. auch Mango, Constantinopolitana, 336.

595 Grundriß siehe Meyer-Plath/Schneider, Landmauer, 97, Abb. 23; Müller-Wiener, Topographie, 244, Abb. 274

596 Meyer-Plath/Schneider, Landmauer, Taf. 45b.

597 Meyer-Plath/Schneider, Landmauer, 107 (Meyer/Plath).

598 Van Millingen, Walls, Plan of the Palace of the Porphyrogenitus, gegenüber 109. 
Tekfur Sarayı wurde bereits von Van Millingen (1899) mit den erstmals von Georgios Pachymeres und später von Ioannes Kantakuzenos erwähnten tô̂

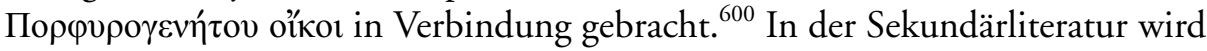
der Palast bzw. sein Vorgänger zwar zwischen dem 9. und 14. Jahrhundert datiert, ${ }^{601}$ doch blieb die Frage offen, ob er auch als Residenz der palaiologischen Kaiser gedient hatte. Es wird nämlich zumeist angenommen, daß der Blachernen-Palast die einzige und letzte Residenz der Kaiser war. ${ }^{62}$ Bereits Mango hat dies bezweifelt: ${ }^{603}$

What is rather less clear is whether Tekfursarayı served as the imperial palace in the last decades of Byzantine history or whether the emperor continued to reside in the old palace of Blachernae. The first alternative appears to me the more likely.

Aus frühpalaiologischer Zeit erfahren wir, daß Kaiser Michael VIII. Palaiologos nach der Rückeroberung der Stadt (1261) sich in dem am Marmarameer gelegenen alten Palast niederließ, da die im Blachernen-Viertel befindliche Anlage in sehr schlechter Verfassung war. ${ }^{604}$ Obwohl der Blachernen-Palast während der Regierungszeit dieses Kaisers wieder in einen bewohnbaren Zustand versetzt wurde, erfährt man aus Reiseberichten des 15. Jahrhunderts, daß er um diese

599 Historische Fotografien, 42. - Die beiden Fotografen Pascal Sébah und Policarpe Joaillier signierten zwischen 1888 und Anfang des 20. Jahrhunderts gemeinsam, siehe ebd., 10; vgl. auch Özendes, Sébah ve Joaillier, 480-481.

600 Kantakuzenos, I, 305/21; vgl. Van Millingen, Walls, 109-110. - Auf die Erwähnung durch Pachymeres II, 161/8-9 (Pachymèrés, Relations, IV, 179/25-26) machte zuerst Kidonopoulos, Bauten, 167, aufmerksam.

601 Für eine Liste der verschiedenen Datierungen bis zum Jahr 1943 siehe Meyer-Plath/ Schneider, Landmauer, 95-96 (Schneider); ebd.98, bringt Schneider den in den Quellen erwähnten Porphyrogennetos mit Konstantin, dem dritten Sohn Michaels VIII. Palaiologos, in Verbindung. Mango, Constantinopolitana, 334: „If as A. M. Schneider argues, the Porphyrogenitus in question was Constantine, third son of Michael VIII, then the building ought to be dated before 1291, when this prince was imprisoned for the rest of his unhappy life. A date between 1261 and 1291 is, I believe, historically very likely." So auch Kidonopoulos, Bauten, 168. Müller-Wiener, Topographie, 244, hält eine Datierung „im Zusammenhang mit dem Ausbau des Blachernenpalais 1261/71 (?)“ für möglich. Für eine Datierung des Palastes, ausgehend von stilistischen Merkmalen, um 1300 siehe Restle, Istanbul 306; in das erste Quartal des 14. Jahrhunderts bei Krautheimer, Architecture, 448; nach 1350 bei Velenis, Hermeneia, 102-103, 163-165, und Ousterhout, Developments, 79. - Zu Konstantinos Porphyrogennetos siehe PLP 9, 21492, wo seine Lebensdaten zwischen 1261-1306 angegeben werden.

602 Siehe z. B. Meyer-Plath/Schneider, Landmauer, 96 (Schneider); vgl. Setton, Papacy, 125: Hier wurde - wie schon von früheren Autoren - Sphrantzes zitierend erwähnt, daß der Kaiser unmittelbar vor der Eroberung von der Hagia Sophia zum Blachernen-Palast

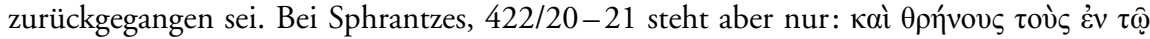
$\pi \alpha \lambda \alpha \tau i ́ \omega$ (ohne Erwähnung eines bestimmten Palastes).

603 Mango, Constantinopolitana, 336; ähnlich auch Müller-Wiener, Topographie, 244. 604 Vgl. Anm. 594. 
Zeit wiederum abermals baufällig gewesen sein muß. Eine klare Auskunft über die Zustand gibt uns der spanische Gesandte Ruy Gonzáles de Clavijo, der im Winter 1403/04 in Konstantinopel weilte und seinen Empfang durch Kaiser Manuel II. Palaiologos beschrieb: ${ }^{605}$

Am folgenden Tag, dem 1. November, setzten die Gesandten nach Konstantinopel über und fanden, daß Meser Ylario und die anderen aus dem kaiserlichen Haus sie bereits am Tor von Quinigo [Kynegion] erwarteten. Sie bestiegen die Pferde und kamen an eine Kirche, die den Namen Santa Maria de la Cherne [BlachernenKirche] trug. Diese Kirche befand sich im Inneren der Stadt neben einer zur Ruine verfallenen Festung, die den Kaisern als Herberge zu dienen pflegte. Dieses Kastell ließ ein Kaiser zerstören, weil einer seiner Söhne, wie im folgenden erzählt werden soll, sich dort verschanzt hatte.

Der Gesandte berichtet hier zweifelsfrei von den Auseinandersetzungen (13761379) zwischen Ioannes V. und Andronikos IV. ${ }^{606}$ was durch die folgende Passage bestätigt wird: ${ }^{607}$

Derjenige, der jetzt Kaiser von Konstantinopel ist, nennt sich Charmornoly $[\mathrm{Kyr}$ Manuel] was Manuel bedeutet. Sein Bruder war vor ihm Kaiser und hatte einen Sohn, der seinem Vater den Gehorsam verweigerte, so sehr, daß er sogar gegen ihn Stellung nahm. Der türkische Sultan [Murat I.], der Vater dessen, den der TimurBeg besiegte [Bayezıt I.], hatte zu der Zeit ebenfalls einen Sohn, der ihm den Gehorsam verweigerte. Nun verabredeten sich diese beiden Söhne, der des Türken und der des Kaisers, mit dem Ziel, ihre Väter abzusetzen und selbst die Herrschaft zu übernehmen. Auch der Sultan und der Kaiser von Konstantinopel kamen desgleichen überein gegen ihre Söhne und überfielen sie ... Als der Vater frei war, ergriff der Sohn die Flucht. In seiner Erbitterung ließ der Kaiser die Festung zerstören, in der sein Sohn ihn gefangen gehalten hatte, und enterbte ihn.

Nach Meyer-Plath/Schneider spricht der Gesandte hier von der Zerstörung des Anemas-Gefängnisses, das sich möglicherweise in der obersten Etage des Theophilos-Turms 15 (Abb. 143) befand. ${ }^{608}$ Tinnefeld schloß u.a. aus diesem Bericht, daß der Blachernen-Palast unbewohnbar gewesen sei, weshalb Clavijo im Großen Palast empfangen wurde. ${ }^{609}$ In seinem Bericht gibt Clavijos über den Ort des Empfangs jedoch folgende Auskunft: $:^{610}$

Er empfing die Gesandten sehr huldvoll und begab sich mit ihnen in ein Gemach. Sie wurden nun vom Kaiser in einem etwas hohen Empfangsraum mit kleinen

605 Clavijo, Embajada, 134/9; deutsche Übersetzung: Clavijo, Reise, 32.

606 Siehe zuletzt Tinnefeld, Blachernenpalast, 283.

607 Clavijo, Embajada, 140/13; deutsche Übersetzung: Clavijo, Reise, 36-37.

608 Meyer-Plath/Schneider, Landmauer, 101, 102 (Schneider); vgl. Van Millingen, Walls, $149-153$.

609 Tinnefeld, Blachernenpalast, 283: „Man darf annehmen, daß diese Beschädigungen des Blachernenpalastes im Rahmen des Kampfes mit der genuesischen Besetzung 1379 erfolgten."

610 Clavijo, Embajada, 114/10; deutsche Übersetzung: Clavijo, Reise, 22. 
Teppichen begrüßt. Auf einen der Teppiche war das Fell eines Leoparden gelegt, auf dessen Schultern sich ein schwarzes Kissen mit Goldstickerei befand ... Der Kaiser lebte mit seiner Gemahlin, der Kaiserin und drei noch sehr kleinen Kindern zusammen.

Aus dieser Aussage geht m.E. hervor, daß der Kaiser in einer bescheidenen Residenz gelebt hatte. Die weiteren Passagen von Clavijos Bericht lassen vermuten, wo diese Residenz gelegenen haben könnte: ${ }^{611}$

Die Stadt Konstantinopel ist sehr gut geschützt durch eine hohe und starke Mauer mit großen und starken Türmen, die an drei Ecken stehen. Von einer Ecke zur anderen sind es sechs Meilen, so daß der Umfang der ganzen Stadt 18 Meilen oder 6 spanische Meilen beträgt. Zwei dieser Seiten werden vom Meer gebildet und nur die dritte vom Land. An der Ecke, die nicht vom Meer gebildet wird, liegen die Paläste des Kaisers.

Eindeutig spricht der Gesandte hier nicht vom Großen Palast, der am Marmarameer liegt. Nicht nur der von Tinnefeld zu Recht angenommene schlechte Zustand des Blachernen-Palastes, sondern vor allem die präzise Ortsangabe („An der Ecke, die nicht vom Meer gebildet wird“) macht deutlich, daß Clavijo von Tekfur Sarayı spricht. In weiteren Passagen seines Berichts erwähnt er darüber

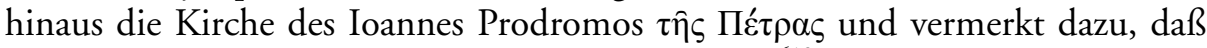
sie sich ganz nahe zum Palast des Kaisers befände. ${ }^{612}$ Der einzige Palast in der Nachbarschaft der bei der Aetios-Zisterne gelegenen Kirche ist Tekfur Sarayı.

Die Aussage Clavijos über den schlechten Zustand des Blachernen-Palastes findet durch weitere Quellen eine Bestätigung, die von Tinnefeld ebenfalls behandelt worden sind, wobei er jedoch Tekfur Sarayı wenig Beachtung geschenkt hat. Ihm zufolge diente der Großen Palast am Marmarameer besonders in den Zeiten, als der Blachernen-Palast in ruinösem Zustand war, als Kaiserresidenz; der Blachernen-Palast sei erst kurz vor 1453 wieder als Residenz benutzt, wie angeblich aus den Quellen hervorgehe. ${ }^{613}$

Mehrere Berichte über die Eroberung von 1453 erwähnen zwar immer wieder eine Palastgegend, die besser zum heutigen Stadtteil Ayvansaray passen würde, wo sich der Blachernen-Palast befand. Damit wird jedoch nicht bewiesen, daß die Kaiser hier noch residierten, obwohl der Ort weiterhin als das eigentliche Palastgebiet angesehen wurde, was letztlich selbstverständlich ist. ${ }^{614}$

611 Clavijo, Embajada, 142/15; deutsche Übersetzung: Clavijo, Reise, 37.

612 Clavijo, Embajada, 172/2; deutsche Übersetzung: Clavijo, Reise, 53. - Vgl. Anm. 457.

613 Tinnefeld, Blachernenpalast, 284.

614 Barbaro (Pertusi, La Caduta I, 23/488-24/490) spricht z. B. von „Chinigio“, also vom byzantinischen „Kynegion“ (Osmanisch „Küngöz“) und macht darauf aufmerksam, daß dieses sich in der Nähe des Palastgebiets befand. Hingegen bringt Tedaldi (Pertusi, ebd., $181 / 21$, Version A, und 181/21, Version B) die Palastgegend mit "Caligaria“ in Zusammenhang, die sicher nicht am Goldenen Horn zu suchen ist (siehe unten Abschnitt 3.4.2.). Es scheint also, daß alle Berichterstatter unter dem Kaiserpalast nicht ein 
Dagegen geben die bildlichen Quellen eine andere Vorstellung über die letzte Residenz der Kaiser: Auf mehreren Kopien der Konstantinopel-Ansicht von Buondelmonti, deren Vorlage aus dem Jahre 1420/22 stammt, ist nur ein einziger Palast mit der Beischrift palatium imperatorum abgebildet (Abb. 28, 44-46). ${ }^{615}$ Wenn auch die einzelnen Zeichnungen zumeist vom tatsächlichen Aussehen des Gebäudes abweichen, ist doch nicht zu bezweifeln, daß schon wegen der Lage des Palastes direkt oberhalb Edirnekapı allein Tekfur Sarayı gemeint sein kann, während an der Nordwestecke der Stadt, also im heutigen Ayvansaray, nur kleine unbedeutende Gebäude und gelegentlich eine Kirche erscheinen.

Über die Lage kurz vor der osmanischen Einnahme geben die Eroberungsberichte von 1453 Auskunft. Eindeutig ist das Gedicht von Ubertino Pusculus, der die Verteilung der byzantinischen Verteidiger an der Landmauer wie folgt schildert: ${ }^{616}$

Aurea porta datur ponto vicina sonanti

Cantacusine tibi, duplici circumdata muro

Andronice, ac geminis hinc inde et turribus altis

castelli in morem conservans limina tuta.

Huic Catarinus adest Venetum de gente vetusta

Contarini, illi parebat multa iuventus.

Credentur, Nicolae, tibi, praefecte, Gudello

cui cognomen erat, Pegaea limina portae.

Haud illo inferior Grittus Baptista fidelis

iungitur huic socius, Venetum decus, optimus illi

fulget in ore nitor fortique pectore virtus.

Armatis micat in mediis magnoque sub armis

corpore circumagitur, sociis fidissima rerum

Spes belli, praesens animoque et corpore tantum est.

bestimmtes Gebäude, sondern ein großes Gebiet verstehen, das vom Goldenen Horn bis

Tekfur Sarayı reichte. In einer anderen Passage seines Berichts sagt Barbaro (bei Pertusi nicht wiedergegeben), daß die Mauer am kaiserlichen Palast weder Zinnen noch Graben hatte. Nach Meyer-Plath/Schneider, Landmauer, 96 (Schneider), spräche dies dafür, daß der Kaiser nicht im Tekfur Sarayı, sondern im Blachernen-Palast residierte. Diese Aussage kann m. E. wieder nur so interpretiert werden, daß Barbaro von der Gegend redet, wo die Palastanlage und die Mauer sich befanden, also von der Strecke, die von Tekfur Sarayı bis zum Goldenen Horn reichte. Auch Pero Tafur (Tafur, 112) macht deutlich, daß das gesamte Gelände immer noch als Kaiserpalast galt. Die Angabe des Diakons Zosima, der 1411, 1419/20 und 1421/22 in Konstantinopel weilte, kann ebenfalls in diesem Sinne interpretiert werden, siehe Majeska, Travelers, 190.

615 Mango, Constantinopolitana, 336; siehe z. B. Barsanti, Buondelmonti, Abb. 61, 64, 6769 und öfter; vgl. auch Effenberger, Buondelmonti, 49, Nr. 39.

616 Pertusi, La Caduta I, 206/151-208/188 (mit italienischer Übersetzung). 
Romani ad portam divi domesticus adstat

Cantacusinus, erat Joannes nomen ab ortu,

Andronicusque, senes ambo, qui gente sub una

Cantacusina orti regis secreta fovebant.

Charsaem servans Lontarius gente Briena

gaudet de socio clara de gente, Fabruci,

Cornaria. Hic Venetus Cretem generosus habebat.

Fide, armis ambo tutantur sorte suprema.

Regia Hieronymo mantadur celsa Minotto

qui Venetis tunc urbe dabat pia iura Pelasga

Huic comes et fidus Joannes scriba Georgus

iunctus adest, civem sibi quem Vincentia premit.

Emmanuel, tibi habenda datur Caligaria civi

praestanti gentis Gudellae sanguine creto.

Ast Xylina tenes, regis de gente vetusta

Palaeologe, prope est portum quae limina portae,

Emmanuel. Longe haud succedit proxima, Graii

quam dicunt Cynagon; Gabriel Trivisanus habebat

cui geminate Venetum parebant forte tririmes.

Hier fällt sofort auf, daß Pusculus die Tore von Süden nach Norden in ihrer richtigen Reihenfolge aufzählt: ${ }^{617}$ Goldenes Tor (Altınkapı), Pege-Tor (Silivrikap1), Romanos-Tor und Charisios-Tor (Sulukulekapı). Doch fehlen einige Tore wie Xylokerkos (Belgratkapı) und Rhesion-Tor bzw. Myriandrion- oder Polyandrion-Tor (Mevlevihanekapi), obwohl diese ebenfalls von tapferen Personen verteidigt wurden. ${ }^{618}$ Auch muß hier angemerkt werden, daß das Johannes-Tor (Edirnekapı) unerwähnt blieb. Unmittelbar nach der Porta Charsea (Sulukulekapı) erwähnt der Autor die regia celsa, den „erhabenen Palast“, auf den folgend er das Caligaria-Tor plaziert. Zorzo Dolfin spricht in seinem fast gleichlautenden Bericht jedoch ausdrücklich von einer porta del palazzo regia und setzt das Tor des Kaiserpalastes ebenfalls zwischen Charisios-Tor und Caligaria-Tor an. ${ }^{619}$ Demnach muß die Porta del Palazzo nördlich vom Charisios-Tor (Sulukulekapı) bzw. nördlich des Johannes-Tors und vor dem Kaligaria-Tor gesucht werden. Die von Pusculus und Dolfin aufgezählten Tornamen geben also eine indirekte Auskunft darüber, daß mit regia celsa bzw. palazzo regia nicht der in Ayvansaray

617 Die Verteilung der byzantinischen Armee wird in mehreren Berichten erwähnt, die mehr oder weniger miteinander übereinstimmen. Im Falle von Pusculus, der Augenzeuge der Eroberung war, ist es wichtig, daß die Stellungen der Verteidiger der Reihe nach aufgezählt werden.

618 So z. B. das Myriandrion von den Gebrüdern Bocchiardi, vgl. Pertusi, La Caduta I, 149/ 22 (Leonardo von Chios); Effenberger, Buondelmonti, 41, 66.

619 Dolfin, 64/54. 
gelegene Blachernen-Palast, sondern das direkt nördlich der theodosianischen Mauer stehende Tekfur Sarayı gemeint ist. ${ }^{620}$

Nicht nur die Schriftquellen, sondern auch ein in dieser Hinsicht wichtiges, wenngleich nicht mehr an seinem ursprünglichen Platz befindliches archäologisches Zeugnis wies darauf hin, daß es sich bei Tekfur Sarayı nur um einen Kaiserpalast gehandelt haben kann: Im einem Fenster des Nordflügels registrierte Mary A. Walker noch 1891 das Palaiologen-Wappen (Abb. 177), daß

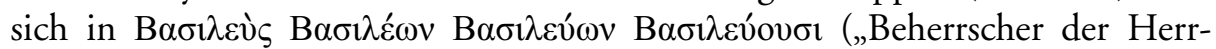
scher, die über die Herrscher herrschen“) auflösen läßt. ${ }^{621}$ Es beweist m.E., daß Tekfur Sarayı nur mit einem regierenden Kaiser in Verbindung gebracht werden kann. Es stellt sich aber die Frage: Seit wann konnte Tekfur Sarayı als Kaiserpalast gedient haben? Im Zusammenhang mit der Belagerung Konstantinopels durch Ioannes Kantakuzenos (1344) erfahren wir von Nikephoros Gregoras, daß der Megas Dux Alexios Apokaukos die gesamte Mauer und die Vormauer zwischen Kaiserpalast und Goldenem Tor repariert und die Mauer - auch des Grabens - mit Zinnen verstärkt hatte. ${ }^{622}$ Der hier erwähnte Kaiserpalast kann nicht mit dem Blachernen-Palast identifiziert werden, da der Mauergürtel mit den Vortürmen und dem Graben sich nur zwischen Goldenem Tor und Tekfur Sarayı erstreckt, ${ }^{623}$ weshalb die Kaiserresidenz keine andere als Tekfur Sarayı

620 Da Porta Charsea, Kaiserpalast und Caligaria-Tor nacheinander erwähnt werden, notierte Van Millingen, Walls, 152: „For both of these writers [Pusculus und Dolfin] place the Gate of Palace between the Gate of Charisius (Edirnè Kapoussi) and the Gate of the Kaligaria (Egri Kapou), and Pusculus describes the palace concerned as 'Regia celsa', an apt description of a building seated, like Tekfour Serai, upon the walls." Wenn man das Charisios-Tor weiterhin mit dem heutigen Edirnekapı identifizieren wollte, würde das nichts an der Tatsache ändern, daß die Porta del Palazzo im Anschluß an die Theodosianische Landmauer gesucht werden muß; vgl. Meyer-Plath/Schneider, Landmauer, 96 (Schneider): „Auch in der regia celsa des Pusculus wird man unschwer die sogenannten 'Y $\psi \eta \lambda$ wiedererkennen dürfen, die einen Teil des eigentlichen Blachernen-Palastes ausmachten. Auf diesem Weg kommt man also nicht weiter."

621 Curtis/Walker, Restes, Abb.67; siehe auch Van Millingen, Walls, 112; Mango, Inscriptions, 54. - Die Platte wurde zu unbekannter Zeit in die Kirche der Panagia $\tau \hat{\eta} s$

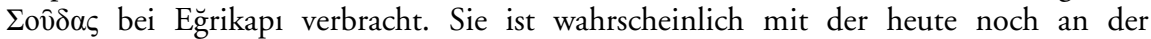
Nordseite des Narthex eingemauerten Platte identisch; vgl. Mango, Inscriptions, 55; zum Motiv siehe Eyice, Boğaziçi, 81-82; zu weiteren Palaiologenwappen ehemals an den Schlußsteinen der unteren Arkaden siehe Mango, Constantinopolitana, 334.

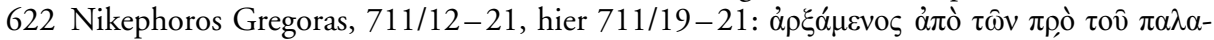

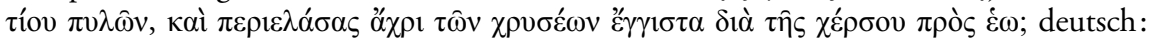
Van Dieten, Nikephoros, III, 123.

623 Ebd.323, Anm.296, identifizierte Van Dieten den erwähnten Palast mit dem Blachernen-Palast; vgl. auch Tinnefeld, Blachernenpalast, 282: „Im Frühjahr 1344 ließ Alexios Apokaukos anläßlich der Belagerung Konstantinopels durch Johannes Kantakuzenos die Landmauer Konstantinopels durch Errichtung einer weiteren Vormauer vom (Blachernen-)palast bis zum Goldenen Horn verstärken." Eine Vormauer an der BlachernenMauer ist archäologisch nicht beweisbar. 
gewesen sein kann. Demnach diente Tekfur Sarayı spätestens 1344 als Palast des Kaisers - wenn auch zunächst nur gelegentlich - und hatte diese Funktion bis zur osmanischen Eroberung beibehalten. ${ }^{624}$ Anhand der Quellenlage ist mit Sicherheit zu sagen, daß sowohl „Tekfur" für „Kaiser“ als auch „Tekfur Baladı“ für „Kaiserpalast“ bereits in frühen osmanischen Quellen vorkommen, ${ }^{625}$ was eindeutig darauf hinweist, daß die Osmanen in der eroberten Stadt einen einzigen damals noch bewohnbaren Kaiserpalast vorgefunden und diesen als „Tekfur Baladı" - also gemäß heutigem türkischen Sprachgebrauch als „Tekfur Saray" - bezeichnet haben.

Die in den späteren Quellen erwähnte porta del palazzo regia muß demnach das Tor an der Westseite des Hofs von Tekfur Sarayı gewesen sein (Abb. 181). Ob Tekfur Sarayı mit dem in den Quellen erwähnten Haus des Porphyrogennetos identisch ist und inwieweit dieses Tor mit dem Porphyrogennetos-Tor in Verbindung gebracht werden kann, läßt sich anhand der wenigen Quellen nicht endgültig klären.

\subsubsection{Das Kaligaria- und das Gyrolimne-Tor}

Außer der Poterne in der Kurtine 2/3, deren Breite 2,40 m beträgt (Abb. 161), befinden sich an der Komnenen-Mauer noch zwei weitere Tore. ${ }^{626}$ Das erste Tor liegt in der Kurtine 6/7; es wird seit dem 15./16. Jahrhundert als Eğrikap1 bezeichnet (Abb. 156, 157) ${ }^{627}$ und bereits im 16. Jahrhundert mit dem Kaligaria-Tor identifiziert. ${ }^{628}$ In der Sekundärliteratur hat sich diese Bestimmung weitgehend eingebürgert, ${ }^{629}$ wobei nur Schneider daran zweifelte. ${ }^{630}$ Das Tor dient noch heute für den Verkehr. Besonders oberhalb des Bogens ist die aufgehende Wand überwiegend restauriert. Ob dieser Zustand unmittelbar auf die Bombardierungen im Jahre 1453 und die nachfolgen Reparaturen zurückzu-

624 Das Jahr 1344 ist nicht weit von dem von Velenis (vgl. Anm.601) angenommenen terminus post quem für den Bau von Tekfur Sarayı entfernt. Falls es sich dabei tatsächlich um das Haus des Porphyrogennetos gehandelt hat, muß Tekfur Sarayı auch mit der Residenz identisch sein, wo Andronikos III. im Jahre 1328 wohnte, was natürlich gegen die Annahme von Velenis spräche.

625 Anonymes Tevârih-i Âli Osman, 108.

626 Meyer-Plath/Schneider, Landmauer, 97, Abb. 23 (Meyer-Plath), notierten zwischen Tekfur Sarayı-Nordturm und Turm 1 der Komnenen-Mauer eine Pforte, die heute nicht mehr zu sehen ist.

627 Fatih Vakfiyeleri, 317, $\$ 17$.

628 Löwenklau, Annales, 479/XII; vgl. MISN-Karte I und II.

629 Vgl. Janin, Constantinople, 283.

630 Meyer-Plath/Schneider, Landmauer, 105 (Schneider); vgl. auch Schneider, Blachernen, 88, Anm. 6. 
führen ist, oder ob das Tor erst nach einem Erdbeben neu gestaltet wurde, bleibt in Dunkeln. ${ }^{631}$

In mehreren europäischen Berichten über die Belagerung von Konstantinopel 1453 wird eine Gegend bzw. ein Tor namens Kaligaria („Schuhmacher“) häufig erwähnt. Die oben zitierten Berichte von Pusculus und Dolfin plazierten dieses Tor hinter dem Palast-Tor (siehe Abschnitt 3.4.1.). Da man im Jahre 1453 unter dem Palast wahrscheinlich nur noch Tekfur Sarayı verstand, müßte das Tor nördlich davon gesucht werden. Kardinal Isidor von Kiev erwähnt in einem Brief an Bessarion, daß ein Tor namens Kaligaria, das besonders schwach war, während der Belagerung durch Theodoros Karstenos verteidigt wurde, der dort auch den Tod fand. ${ }^{632}$ Leonardo von Chios spricht nur von einer KaligariaGegend, wo sich kein Graben befand und die Mauer ziemlich stark gewesen sein soll. $^{633}$ Jacopo Tedaldi gibt uns in dieser Hinsicht eine noch präzisere Auskunft, indem er die Kaligaria-Gegend der Angriffsfront des Karaca Paşa zuzählt und hinzufügt, daß diese Örtlichkeit ganz nahe zum Palast lag. ${ }^{634}$ Von Kritobulos erfuhren wir bereits, daß die Angriffsfront von Karaca Paşa vom Goldenen Horn bis zum Charisios-Tor reichte. ${ }^{65}$ Fast alle Berichte stimmen darin überein, daß in diesem Gebiet bzw. vor dem Tor eine osmanische Kanone stand, während eine zweite vor dem Palast plaziert war, geben aber unterschiedliche Auskünfte über die Stärke der Mauer. Bei der hier aufgestellten Kanone handelte es sich nach Leonardo von Chios um das Riesengeschütz des Kanonenmeisters Urban, das bald zum Romanos-Tor umgesetzt wurde. ${ }^{636}$ Somit besteht kein Zweifel, daß das Tor sich in der Blachernen- bzw. in der Komnenen-Mauer und nahe Tekfur Sarayı befand.

Erstaunlicherweise erwähnen die byzantinischen Quellen kein KaligariaTor: Entweder hatten nur die europäischen Berichterstatter den Namen der Gegend aus Unwissenheit auf ein Tor übertragen, das eigentlich einen anderen byzantinischen Namen hatte, oder die byzantinischen Chronisten haben das Geschehen vor diesem Tor übersehen, was eigentlich nicht möglich erscheint. Unter den byzantinischen Autoren benutzt (Pseudo-)Sphrantzes den Namen

631 Wir wissen aus osmanischen Quellen, daß Eğrikapı bereits 1509 von dem großen Erdbeben betroffen war, siehe Edirne'li Rûhi, 311-312.

632 Pertusi, La Caduta I, 70/76-82. - Nach Schneider, Blachernen, 88, Anm.4, komme Kaligaria im Zusammenhang mit einem Tor nur bei Dolfin vor.

633 Pertusi, La Caduta I, 130/66-68.

634 Pertusi, La Caduta I, $181-182, \$ 21$. - Tedaldi gibt die Entfernung vom Romanos-Tor bis zum Kaligaria-Tor mit ca. zwei Meilen an. Legt man eine italienische Meile mit $1.270 \mathrm{~m}$ zugrunde, so entspräche das ziemlich genau dem Abstand zwischen RomanosTor und Eğrikapı (ca. $2,3 \mathrm{~km}$ ).

635 Siehe Anm. 465.

636 Pertusi, La Caduta I, 134/110-113; siehe auch Anm.360. 
Kaligaria nur zur Bezeichnung einer Gegend. ${ }^{637}$ Er bestätigt die angenommene Lage, indem er darüber Auskunft gibt, daß der Kaiser kurz vor der Eroberung mit seiner Begleitung dort auf einen Turm gestiegen war, von wo aus man einerseits die Tätigkeiten der Belagerer am Goldenen Horn beobachten, andererseits aber den Lärm der osmanischen Armee in der Nähe des Grabens hören konnte. ${ }^{638}$ Demnach befand sich dieser Turm am Südtrakt der KomnenenMauer. ${ }^{639}$ Dort existierten zwei Tore: Die erwähnte Poterne in der Kurtine 2/3 (Abb. 161) und Eğrikapı in der Kurtine 6/7 (Abb. 156, 157). Das zugemauerte Tor in der Kurtine 11/12 (Abb. 148, 158) kann ausgeschlossen werden, da es sich im Nordtrakt befindet und ziemlich weit von Tekfur Sarayı bzw. dem Ende des Grabens der Theodosianischen Landmauer entfernt ist. Somit kommen nur die beiden genannten Tore in Frage, wobei zwei Gründe für Eğrikapı sprechen:

1. Die Poterne in Kurtine $2 / 3$ der Komnenen-Mauer steht in unmittelbarer Nachbarschaft zum Palast (also zum Tor von Tekfur Sarayı). Es wäre ziemlich überflüssig, daß man hier noch eine Kanone aufstellte, zumal bereits eine vor dem Palast plaziert war.

2. Die in dieser Gegend bzw. vor dem Tor aufgestellte Kanone wurde später von dort zum Romanos-Tor transportiert, da sie hier nicht sehr erfolgreich war. Das bedeutet wiederum - wenn die Aussage zutrifft -, daß diese Kanone nicht in der Nachbarschaft der anderen vor dem Palast gestanden haben kann. Die Kanone war wahrscheinlich deswegen nicht erfolgreich, weil sie an einem abschüssigen Gelände stand, wofür nur der Bereich vor Eğrikapı in Frage kommt.

Das zugemauerte Tor in der Kurtine 11/12 ist mit dem Gyrolimne-Tor in Verbindung gebracht worden (Abb. 148, 158) ${ }^{640}$ Ein Ort namens Gyrolimne (Silber-See) kommt u. a. bei Niketas Choniates vor, ${ }^{641}$ jedoch ohne Verbindung mit einem Tor. Es wird zu Recht angenommen, daß der Name Gyrolimne sich auf eine Bucht des Goldenen Hornes bezieht. ${ }^{642}$ Erst aus spätbyzantinischen Quellen, vor allem von Ioannes Kantakuzenos, erfahren wir, daß ein Gyroli-

637 Sphrantzes, 422/27. - In der deutschen Übersetzung wird es als „Kaligaria-Tor“ wiedergegeben, siehe Ivánka, Chronikon, 74. Der Name Kaligaria kommt in den Quellen bereits im 14. Jahrhundert vor, siehe Delehaye, Typika, 93.

638 Sphrantzes, 422/27.

639 Lediglich im Wiener und Cambridger Torverzeichnis wird irrtümlich das Kynegion-Tor an der Seemauer des Goldenen Horns als Kaligaria-Tor bezeichnet, vgl. Effenberger, Buondelmonti, Tabelle III, Nr.3A.

640 Mordtmann, Esquisse, 37; Van Millingen, Walls, 126-127. - Meyer-Plath/Schneider, Landmauer, 105, zufolge befindet sich dieses zugemauertes Tor an der von Ioannes V. Palaiologos reparierten Kurtine, d.h. an der Kurtine 12/13 (Schneider), während MeyerPlath (114) es an die richtige Stelle - also an die Kurtine 11/12 - versetzte.

641 Niketas Choniates, 543/95-7; siehe Janin, Constantinople, 355.

642 Van Millingen, Walls, 126-127. 
mne-Tor eigentlich kaiserlichen Zwecken diente und der Kaiser durch dieses Tor öfter zur Jagd ausritt. ${ }^{643}$ Weiterhin geht aus diesen Berichten hervor, daß das Tor sich in unmittelbarer Nähe des Blachernen-Palastes befand. ${ }^{644}$ Obwohl die Quellenlage sehr spärlich ist, hat bereits Van Millingen das Gyrolimne-Tor mit der Toranlage in der Kurtine 11/12 der Komnenen-Mauer identifiziert. ${ }^{645}$ Seine Gleichsetzung basierte nicht zuletzt auch auf drei kaiserlichen Büsten, die den Torbogen schmücken (Abb. 159a, b, c), und auf den Berichten, die das Tor mit der kaiserlichen Jagd in Verbindung bringen. Es befand sich nämlich außerhalb des Blachernen-Viertels eine kaiserliche Jagd- und Vergnügungsanlage, das Philopation, das durch eines von den Toren des Blachernen-Viertels erreichbar gewesen sein muß. ${ }^{646}$ Meyer-Plath/Schneider lehnten diese Identifizierung mit der Begründung $\mathrm{ab}$, daß die Bezeichnung $\pi \dot{\prime} \lambda \eta$ sich nur mit einem stattlichen Tor in Verbindung bringen lasse, wofür allein Eğrikapı in Betracht komme. ${ }^{647}$ Da das Tor in der Kurtine 11/12 3,70 m breit ist und - obwohl es mindestens $2 \mathrm{~m}$ im Schutt steht - sehr hoch gewesen sein muß, erweist es sich als größer als Eğrikapı, dessen Breite nur $3 \mathrm{~m}$ beträgt. Die Identifizierung des zugemauerten Tors in Kurtine 11/12 mit dem Gyrolimne-Tors der Komnenen-Mauer sollte also beibehalten werden, solange keine besseren Argumente für einen Gegenvorschlag vorgebracht werden können. Doch die an diesem Tor angebrachten drei Büsten verdienen eine zusätzliche Betrachtung.

\subsubsection{Die Büsten am Gyrolimne-Tor}

Die mittlere und die rechte der drei Büsten haben bereits ihre Köpfe verloren. Beide Büsten sind mit einer Dalmatika und einem Perlenkragen bekleidet. Der Perlenkragen der mittleren besteht aus je einer doppelten Perlenreihe unten und oben sowie einem abwechselnd mit rechteckigen Edelsteinen und vier im Quadrat angeordneten Perlen geschmückten mittleren Teil. Am unteren Saum hängen Pendilien mit Perlen herab (Abb. 159a). Bei der rechten Büste besteht der Mittelteil des Kragens nur aus einer Reihe Cabochons (Abb. 159b). Van Millingen hat die mittlere Büste am Ende des 19. Jahrhunderts noch in besserem Zustand mit fast vollständig erhaltenem Kopf gesehen und am Anfang seines Buches abgebildet (Abb. 159c). Der Abbildung ist zu entnehmen, daß die Figur eine Haubenkrone trug. Erkennbar sind noch eine Perlenreihe über der

643 Kantakuzenos I, 89/23-90/1; deutsche Übersetzung: Fatouros/Krischer, Geschichte, I, 69.

644 Kantakuzenos I, 289/4-5; deutsche Übersetzung: Fatouros/Krischer, Geschichte, I, 169.

645 Van Millingen, Walls, 127.

646 Quellen und Diskussion zu dieser Jagdanlage bei Maguire, Gardens, 251-264.

647 Meyer-Plath/Schneider, Landmauer, 105 (Schneider); 114 (Meyer-Plath) wird die Breite des Tors mit 3,03 m angegeben: vgl. auch Schneider, Blachernen, 88. 
Stirn und kreuzweise angeordnete Perlen ein wenig darüber. Der vom Kopf ausgehende hintere Teil der Kopfbedeckung (?), von dem noch ein Stück erhalten ist, war möglicherweise die rückseitige Stütze. Die Haare scheinen nach hinten aufgebunden zu sein. Meyer-Plath hat die Büste nur kurz erwähnt. ${ }^{648}$

Die Figuren, vor allem die mit Pendilien und Perlen, lassen sich mit denen einiger Kaiserinnen des 6. Jahrhunderts vergleichen. Einen etwas bescheideneren Perlenkragen trägt die Ariadne-Büste auf einem Kapitell in İstanbul. ${ }^{649}$ Auf den beiden Diptychontafeln in Florenz und Wien, die um 500 datiert werden, ist die Kaiserin mit einer Chlamys bekleidet und mit einem etwas anderen Perlenkragen geschmückt. ${ }^{650}$ Die gleiche Kaiserin (Ariadne) ist im oberen Register des Clementinus-Diptychons in Liverpool aus dem Jahre 513 als Büste mit einem ähnlichen Perlencollier wiedergegeben. ${ }^{651}$ Sie korrespondiert hier mit einer Büste des Kaisers Anastasius, der ebenfalls in einem Medaillon erscheint und mit einer Chlamys bekleidet ist. ${ }^{652}$ Der Perlenkragen gehörte auch in justinianischer Zeit zum Kaiserinnengewand. Einen solchen trägt die Kaiserin Theodora auf dem Mosaik in San Vitale. ${ }^{653}$ Dieser weist mit demjenigen unserer Büsten große Ähnlichkeit auf und zeigt auch die mit angehängten Perlen geschmückten Pendilien. An den Stadtmauerbüsten sind jedoch keine Diadembzw. Kronhauben mit herabhängenden Pendilien mehr zu erkennen. Anhand der Vergleichsbeispiele und des Haarstils (Foto von Van Millingen, Abb. 159c) sind die beiden einigermaßen erhaltenen Büsten als weibliche Figuren zu bestimmen. ${ }^{654}$ Der Perlenkragen läßt sich mit den Beispielen aus dem 6. Jahrhundert vergleichen. Auch das Fehlen des Loros, der seit mittelbyzantinischer Zeit fester Bestandteil des Kaiserornats ist, spricht für eine Datierung der Kaiserinnen in das 6. Jahrhundert. Es handelt sich bei den Stücken also um Spolien, die jedoch im Rahmen eines durchdachten Konzepts hier eingefügt worden sind. Schon aus diesem Grund muß dem Tor eine besondere Bedeutung beigemessen worden sein.

648 Meyer-Plath/Schneider, Landmauer, 114.

649 Firatli, Sculpture 116-117, Nr.217, Taf.69, Abb. 217.

650 Siehe dazu, Volbach, Elfenbeinarbeiten, 49, Nr. 51; 50, Nr. 52; Taf. 27, Abb. 51 und 52.

651 Volbach, Elfenbeinarbeiten, 35-36, Nr. 17, Taf.7, Abb. 15; siehe auch Bühl, Constantinopolis, 197-198, Abb. 103; ein weiteres Bespiele ebd. 199, Abb. 199.

652 Für weitere Beispiele siehe Vollbach, Elfenbeinarbeiten, Taf. 8 und 9.

653 Für eine Detailaufnahme siehe Deichmann, Ravenna, Abb. IX.

654 Siehe dazu Emmanuel, Hairstyles, 113-119. 